\title{
Un Esquema Numérico para la Aproximación de Boussinesq en Medios Porosos
}

\author{
Tesis \\ que para obtener el grado de Maestra en Matemáticas \\ presenta \\ Elsa Báez Juárez \\ bajo la dirección del Dr. Alfredo Nicolás Carrizosa
}

Departamento de Matemáticas

Universidad Autónoma Metropolitana-Iztapalapa 


\title{
Un Esquema Numérico para la aproximación de Boussinesq en Medios Porosos
}

\author{
TESIS \\ que para obtener el grado de Maestra \\ en Matemáticas presenta \\ Elsa Báez Juárez \\ bajo la dirección del Dr. Alfredo Nicolás Carrizosa
}


A mi hijo Francisco

Al Dr. A. Noé Morales D.

A mi madre Elsa y a mis hermanos: Jorge, Graciela, Alejandro, Claudia, Juan Carlos y Gabriela

A la memoria de mi padre y de mi hermano Juan 


\section{AGRADECIMIENTOS}

Agradezco a mi asesor, Dr. Alfredo Nicolás Carrizosa, por la guía, esfuerzo, tiempo y paciencia que me dedicó en el desarrollo de la tesis; a la Dra. Patricia Saavedra Barrera, al Dr. Carlos Ortíz Alemán y al Dr. Francisco J. Sánchez Bernabe por los comentarios y correcciones hechas a la presente tesis.

De igual manera agradezco al CONACYT por el apoyo económico que recibí durante el desarrollo de mis estudios de maestría. 


\section{RECONOCIMIENTO}

La autora de esta tesis y el asesor deseamos agradecer de manera especial al Dr. Alen Díaz Cárdenas, profesor del area de Termofluidos del Departamento de Energía de la UAM-A, pues su experiencia y conocimientos sobre el tema lo condujeron a sugerirnos el presente problema. 


\section{Contenido}

1 Introducción 3

2 Relaciones de la Mecánica de Fluidos en Medios Porosos 8

2.1 Introducción . . . . . . . . . . . . . . 8

2.2 La Ecuación de Continuidad . . . . . . . . . . . . . . . 9

2.3 La Ecuación de Movimiento . . . . . . . . . . . . . . 10

2.4 La Ecuación de Energía . . . . . . . . . . . . . . . . . 12

2.5 Dinámica de Fluidos aplicada a Medios Porosos . . . . . . . 13

2.6 Ley de Darcy . . . . . . . . . . . . . . . . . . . . . . 14

2.7 Aproximación de Boussinesq . . . . . . . . . . . . 15

2.8 Condiciones Iniciales y de Frontera . . . . . . . . . . . 17

3 Problema Continuo 18

3.1 Introducción . . . . . . . . . . . . . . . 18

3.2 Simplificación de la ecuación de movimiento vía función de corriente . . . . . . . . . . . . . . . . . 19

3.3 Problema Adimensional . . . . . . . . . . . . . . . 21

4 Problema Discreto 24

4.1 Introducción . . . . . . . . . . . . . . . . 24

4.2 Problema Semidiscreto (Discretización en Tiempo) . . . . . 25

4.3 Método de Solución para el Problema Estacionario . . . . . 26

4.4 Discretización en el espacio . . . . . . . . . . . . . . 28

4.5 Número de Nusselt . . . . . . . . . . . . . . . . . . . . . 30

5 Resultados Numéricos 32

5.1 Introducción . . . . . . . . . . . . . . . 32

5.2 Condiciones de Frontera Dirichlet . . . . . . . . . . . . 33 
5.3 Condiciones de Frontera Mixtas . . . . . . . . . . . . . 44

6 Conclusiones $\quad 47$

6.1 Condiciones de Frontera Dirichlet . . . . . . . . . . . . . . . . 47

6.2 Condiciones de Frontera Mixtas . . . . . . . . . . . . . . . 48

6.3 Sugerencias para la Resolución de Problemas Similares . . . . 50

A Variables dimensionales y adimensionales $\quad 52$

B Método Iterativo SOR por Bloques 54

$\begin{array}{ll}\text { C Fishpack } & 57\end{array}$ 


\section{Capítulo 1}

\section{Introducción}

Resumen: Se presenta una breve reseña de algunos antecedentes sobre el estudio de la transferencia de calor en medios porosos, posteriormente se hace una descripción del trabajo a realizar así como de los objetivos a alcanzar en el mismo.

Cuando hablamos de flujos en medios porosos nos referimos a fluidos (líquidos o gaseosos) que se muever a través de un material sólido el cual se caracteriza, en términos generales, por tener gran cantidad de pequeños poros, algunos de los cuales están interconectados. Este tipo de flujo que se presenta con frecuencia tanto en la naturaleza como en algunas áreas de la industria, y tiene diversas aplicaciones prácticas de primordial importancia para el hombre, razón por la cual analizar y entender la mecánica asociada a estos sistemas es de gran interés en muchas ramas de la ciencia y de la ingeniería.

Estos procesos se encuentran por ejemplo en la naturaleza, en donde los organismos vivos son todos porosos, las funciones que los mantienen vivos no serían posibles sin estos poros. Los poros hacen posible la respiración así como la circulación de los fluidos naturales, tanto en plantas como en animales. La estructura porosa de la piel de los animales también sirve para aislar el calor.

En el mundo inanimado la estructura porosa es igualmente amplia e importante. El suelo es poroso al igual que las rocas naturales y el grafito. Entre los productos industriales, los materiales porosos son también numerosos y de gran valor práctico. Los materiales de construcción tales como la cerámica, 
el concreto y la madera son porosos. Nuestra ropa está hecha de textiles porosos; asimismo, el papel está hecho de fibras y es también poroso.

Por otra parte, estos fenómenos juegan un papel preponderante en el desarrollo de colectores solares y de sistemas de enfriamiento para reactores nucleares; en los campos de la mecánica e hidrología de los suelos; en geofísica y en ciencias de la tierra se relacionan, por ejemplo, con la transferencia de calor que se da tanto en las profundidades de la Tierra como en las capas superficiales de regiones geotérmicas y con la extracción de petróleo y de gas natural.

En este último caso, los depósitos de gas o petróleo son una formación geológica porosa que contiene en su espacio poroso, además de agua, al menos un hidrocarburo (gas o petróleo). Los yacimientos, ricos en materia orgánica a partir de los cuales se originan los hidrocarburos, se llaman yacimientos fuente. En estos yacimientos, los hidrocarburos se crean en la forma de un gran número de minúsculas burbújas rodeadas por el agua que ocupa el espacio poroso. A partir de los yacimientos fuente, los hidrocarburos emigran hacia los depósitos rocosos donde se acumulan en cantidades tales que son de gran interés comercial.

Las principales fuerzas responsables de esta migración de hidrocarburos son las de capilaridad y las de flotación (empuje hacia arriba). Tanto el petróleo como el gas son más ligeros que el agua que los rodea en el espacio poroso de las rocas, por lo cual ésta última tiende a ir hacia el fondo y a empujar a los hidrocarburos hacia arriba (fuerza de flotación). Por lo tanto, estas fuerzas de flotación conducen a la separación de las capas de agua, petróleo y gas en el espacio poroso de los depósitos rocosos. Para permitir la acumulación, la migración de hidrocarburos asciende hacia yacimientos más elevados a través del medio poroso provisto por las formaciones geológicas, y finalmente se detiene debido a una capa de material impermeable o (casi impermeable) que sirve como un tope superior a los mantos de hidrocarburos, de donde son posteriormente extraídos.

Ahora bien, para desarrollar una representación cuantitativa que describa el comportamiento de fluidos a través de medios porosos y permita su análisis y estudio, es necesario primero establecer los principios físicos que lo determinan. Sin embargo, no obstante el hecho de despreciar las fuerzas inerciales (debidas a la presencia de pequeñas velocidades características de los flujos en medios porosos) y de que el desarrollo de mejores computadoras digitales ha permitido analizar con mayor detalle los efectos no lineales y los fenómenos de acoplamiento que surgen en estos casos, aún son muchas las dificultades 
matemáticas que se presentan al aplicar estas ecuaciones a medios porosos.

En este sentido, la mayor parte de las investigaciones realizadas involucran generalmente sistemas bidimensionales, en donde con frecuencia se propone un dominio rectangular, ya que son más sencillos de resolver mediante las técnicas de análisis conocidas; ocasionalmente se han estudiado problemas tridimensionales y aún en estos casos se hace la simplificación al caso bidimensional correspondiente.

Una vez que se ha planteado el modelo matemático a resolver, uno de los aspectos de mayor interés en muchos de estos problemas, y que por tanto recibe más atención es el proceso de transferencia de energía. Entre estos procesos se encuentra la transferencia de calor por convección natural, la cual sucede cuando un fluido en un medio poroso se pone en movimiento debido a las diferencias de densidad que se producen por los gradientes de temperatura.

En algunos estudios, se encontró que existen dos parámetros básicos (en el problema adimensional) que determinan la evolución de este tipo de problemas, uno de ellos es la relación entre el largo y ancho del dominio rectangular (razón geométrica) y el otro es el número de Rayleigh $(R)$ el cual expresa el balance entre la fuerza de flotación y los procesos difusivos, los cuales retardan el movimiento y tienden a estabilizarlo. Es por ello que en diversas investigaciones se hace énfasis particular sobre los valores que tomarán estos parámetros en el modelo a considerar.

Algunos de los primeros en tratar de analizar el flujo de fluidos en medios porosos fueron Horton y Rogers en 1945 y Lapwood en 1948; a partir de éstas y de otras investigaciones se har ido aclarando, entre otras cosas, los diversos mecanismos en que se desarrolla la transferencia de calor en un fluido contenido en un medio poroso.

Por ejemplo, Batchelor [1] demostró que para números de Rayleigh pequeños, así como para valores grandes de la razón geométrica, la principal forma de tranferencia de calor es por conducción. Mientras que Gill [2] consideró el caso en que la razón geométrica era fija pero con números de Rayleigh grandes encontrando que la convección dominaba el proceso de transferencia de calor.

J. W. Elder [3] analizó el flujo convectivo en un medio poroso mediante técnicas de análisis numérico, mismas que corroboró experimentalmente. Su objetivo principal fue determinar la relación existente entre el número de iteraciones de las ecuaciones estacionarias con el tiempo requerido por las ecuaciones no estacionarias respectivas, para los cuales ambas soluciones al- 
canzan detalles semejantes.

Cormack, Leal e Imberger [4] determinaron que fijando el número de Rayleigh y para una razón geométrica suficientemente pequeña, nuevamente la conducción era el modo dominante de transferencia de calor.

R. N. Horne y M. J. O'Sullivan [5] estudiaron la estabilidad de flujos de convección natural en un medio poroso confinados en un dominio rectangular calentado en su frontera inferior tanto uniforme como no uniformemente. Encontraron que en el caso de calentamiento uniforme el flujo era conducido a un estado estacionario o a uno fluctuante. Sin embargo, en el caso en que existía una considerable no uniformidad en el ingreso de calor, el tipo de flujo que se obtenía era oscilatorio, siempre que el número de Rayleigh fuera suficientemente grande.

G. E. Schubert y J. M. Straus [6] corroboraron los resultados obtenidos por Horne y O'Sullivan para el caso de calentamiento uniforme; la aparición de uno u otro flujo dependía del movimiento original en la región, lo cual denotaba un ejemplo de la dependencia de los flujos estudiados sobre las condiciones iniciales.

Por su parte D. M. Christopher [7] y [8] realizó estudios para flujo en medios porosos, conducidos por convección natural, manteniendo fija la razón geométrica y para números de Raleigh entre 1,000 y 10,000. Propone un esquema numérico de diferencias finitas, de cuarto orden, para resolver el sistema de ecuaciones que obtiene y analiza las diversas soluciones que obtiene al variar las condiciones iniciales para la temperatura.

Nuestro trabajo se basa principalmente en los artículos citados anteriormente de D. M. Christopher: proponemos un esquema numérico alternativo para resolver las ecuaciones acopladas de momento (ecuación de momento en términos de la función corriente) y energía que resultan de modelar la convección natural de un fluido en un medio poroso para números de DarcyRaleigh de 1,000 y 10,000 con condiciones de frontera de tipo Dirichlet y, posteriormente, mixtas para la temperatura, manteniendo siempre condiciones Dirichlet para la función de corriente. Asimismo hacemos un análisis comparativo de los resultados obtenidos por él y los presentados por nosotros ya que una vez que Christopher plantea el sistema de ecuaciones a resolver, utiliza un esquema de diferencias finitas centradas de cuarto orden para la discretización espacial y el esquema de Cranck-Nickolson para la discretización del término dependiente del tiempo, y resuelve la ecuación de movimiento utilizando la distribución de temperatura calculada en el paso de tiempo anterior, al desacoplar las ecuaciones en el tiempo; mientras que en nuestro esquema 
numérico, usamos un método de diferencias finitas de segundo orden para la discretización en espacio y el método de Gear para la discretización temporal ( 20 . orden en exactitud), y mantenemos el acoplamiento, en tiempo, del modelo mediante un esquema iterativo.

La manera en que resolvemos el problema no-lineal estacionario de ecuaciones elípticas acopladas (una lineal y la otra no-lineal) que se obtiene de la discretización implícita en el tiempo es mediante un proceso iterativo de punto fijo, con lo cual en cada iteración resolvemos problemas elípticos desacoplados, llegando posterormente a obtener sistemas de ecuaciones algebraicas lineales. Estos ltimos pueden ser resueltos aplicando un método apropiado de algebra lineal numérica para obtener la solución final del problema en una computadora.

En resumen, en el presente trabajo estudiaremos la convección natural de flujos en medios porosos en donde los objetivos a alcanzar son:

1. Resolver numéricamente el problema planteado anteriormente, así como describir y comparar los puntos de coincidencia y las diferencias que se presenten entre los resultados que obtengamos en este trabajo, con los presentados por Christopher $[7,8]$.

2. Basándonos en los resultados obtenidos para condiciones de frontera de tipo Dirichlet para la temperatura, resolver el mismo problema considerando ahora condiciones de frontera de tipo mixto para la temperatura. Este último tipo de condiciones mixtas, no fueron reportadas por Christopher, y son, en consecuencia, resultados nuevos.

3. Describir de manera breve, cómı mediante modificaciones relativamente sencillas al modelo, este método se puede aplicar para resolver problemas similares con diversas condiciones de frontera. 


\section{Capítulo 2}

\section{Relaciones de la Mecánica de Fluidos en Medios Porosos}

Resumen: Se presentan las ecuaciones que rigen el movimiento de un fluido en general. A continuación se consideran la modificaciones que sufren algunas de estas ecuaciones en medios porosos, considerando que se satisfacen la Ley de Darcy y la aproximación de Boussinesq.

\subsection{Introducción}

Hasta ahora han resultado suficientes tres leyes físicas fundamentales para estudiar, en general, el flujo de fluidos, independientemente de su naturaleza, y que expresadas en términos matemáticos adecuados, describen un fluido en movimiento. Estas leyes se listan a continuación, al igual que la designación de sus formulaciones matemáticas.

1. Ley de conservación de la materia $\rightarrow$ ecuación de continuidad,

2. Segunda ley de Newton del movimiento $\rightarrow$ ecuación de movimiento, y

3. Primera ley de la termodinámica $\rightarrow$ ecuación de energía.

Además de estas leyes, también son necesarias otras relaciones auxiliares, tales como la ecuación de estado, que caracteriza tanto la naturaleza como la situación termodinámica del fluido en consideración. 
Una vez planteadas las ecuaciones indicadas anteriormente, y que se aplican a cualquier sistema de flujo, veremos cómo se transforman estas relaciones para caracterizar el flujo de fluidos a través de medios porosos.

\subsection{La Ecuación de Continuidad}

La ley de conservación de la materia establece que la masa del fluido en cualquier sistema cerrado no se crea ni se destruye. La ecuación correspondiente se deduce aplicando un balance de materia a un elemento unitario de volumen a través del cual está circulando el fluido; entonces, la velocidad de acumulación de materia debe ser igual a la diferencia entre la velocidad de entrada de materia y la velocidad de salida de materia. Esto es equivalente a la ecuación

$$
\frac{\partial}{\partial x}\left(\rho v_{x}\right)+\frac{\partial}{\partial y}\left(\rho v_{y}\right)+\frac{\partial}{\partial z}\left(\rho v_{z}\right)+\frac{\partial \rho}{\partial t}=0,
$$

donde $\mathrm{v}$ es el vector velocidad del fluido en la posición $(x, y, z)$ con componentes $\left(v_{x}, v_{y}, v_{z}\right)$; y $\rho$ es la densidad del fluido en el mismo punto.

La ecuación anterior se puede reescribir en forma compacta como

$$
\nabla \cdot(\rho \mathbf{v})+\frac{\partial \rho}{\partial t}=0
$$

la cual se conoce como ecuación de continuidad. Sin embargo, debemos especificar, además, la naturaleza del fluido que se está involucrando y el carácter termodinámico de su flujo. Esto es, debemos conocer la ecuación de estado que define al fluido y la cual es, en términos generales, una relación entre su temperatura, su presión y su densidad.

En una gran cantidad de fluidos, la densidad puede ser considerada constante durante todo su movimiento (tanto en espacio como en tiempo). En otras palabras, no hay compresión o expansión notables del fluido; en tales casos se habla de fluidos incompresibles, lo cual implica que $\rho=$ cte., por lo que la ecuación (2.2) se reduce a

$$
\nabla \cdot \mathrm{v}=0
$$

Esta ecuación puede considerarse como la condición sobre la distribución de velocidad en cualquier sistema de fluido incompresible. Analíticamente, sin embargo, no es suficiente para determinar las componentes individuales 
de la velocidad, y físicamente no se discrimina entre un fluido incompresible y otro, ni se distingue entre sistemas expuestos a fuerzas externas, tales como la gravedad, y aquellos flujos que sólo están bajo la acción de diferencias de presión, o entre fluidos que fluyen a través de medios porosos y fluidos que fluyen por conductos no obstruidos.

Entonces, debemos caracterizar el fluido y establecer explícitamente cómo reacciona al gradiente de presión y a las fuerzas externas. Específicamente, debemos formular la equivalencia hidrodinámica a la Segunda Ley de Newton, es decir, la ecuación de movimiento. Los detalles de esta formulación dependerán de la naturaleza del fluido y de las condiciones bajo las cuales fluye.

\subsection{La Ecuación de Movimiento}

Considerando un elemento unitario de volumen, encontramos que en general estará sujeto a tres tipos de fuerzas: los gradientes de presión, fuerzas externas (como la debida a la gravedad) y fuerzas que se oponen al movimiento del fluido y que se deben a la resistencia interna o fricción que experimenta el fluido.

La ecuación dinámica del movimiento se obtiene al igualar la suma de estas fuerzas al producto de la masa y la aceleración del elemento de volumen al cual se aplica la fuerza (lo cual no es más que la segunda ley de Newton). Debido a que la velocidad en el fluido variará, en general, de punto a punto, debe hacerse notar en los cálculos su aceleración, que no sólo hará que la velocidad del elemento del fluido cambie durante cualquier intervalo de tiempo en la posición que tenía originalmente, sino que experimentará un cambio adicional causado por el hecho de que en el intervalo de tiempo se ha movido a otra región del fluido. La aceleración debe, por lo tanto, expresarse mediante la derivada temporal total de la velocidad

$$
\mathbf{a}=\frac{d \mathbf{v}}{d t} \equiv \frac{\partial \mathbf{v}}{\partial t}+\frac{d x}{d t} \frac{\partial \mathbf{v}}{\partial x}+\frac{d y}{d t} \frac{\partial \mathbf{v}}{\partial y}+\frac{d z}{d t} \frac{\partial \mathbf{v}}{\partial z},
$$

donde los cambios infinitesimales de posición de una partícula están directamente relacionados con la velocidad local, es decir

$$
d x=v_{x} d t \quad d y=v_{y} d t \quad d z=v_{z} d t .
$$


Sustituyendo estos valores en la ecuación (2.4) obtenemos la expresión para la aceleración de la partícula

$$
\frac{d \mathbf{v}}{d t}=\frac{\partial \mathbf{v}}{\partial t}+\left(v_{x} \frac{\partial \mathbf{v}}{\partial x}+v_{y} \frac{\partial \mathbf{v}}{\partial y}+v_{z} \frac{\partial \mathbf{v}}{\partial z}\right)
$$

El primer término del segundo miembro se denomina derivada (o aceleración) local, el cual indica el cambio en la velocidad durante un tiempo infinitesimal $d t$ en un punto fijo en el espacio, y se anula cuando el flujo es estacionario, esto es, independiente del tiempo. Los tres términos entre paréntesis forman la derivada (o aceleración) convectiva, la cual aparece cuando la partícula se mueve a través de regiones en que la velocidad varía. La aceleración convectiva puede ser expresada como

$$
v_{x} \frac{\partial \mathbf{v}}{\partial x}+v_{y} \frac{\partial \mathbf{v}}{\partial y}+v_{z} \frac{\partial \mathbf{v}}{\partial z} \equiv(\mathbf{v} \cdot \nabla) \mathbf{v},
$$

en esta forma, podemos escribir la aceleración total, ec. (2.6) de manera breve como

$$
\mathbf{a}=\frac{d \mathbf{v}}{d t}=\frac{\partial \mathbf{v}}{\partial t}+(\mathbf{v} \cdot \nabla) \mathbf{v}
$$

Notamos que aunque el término $(\mathbf{v} \cdot \nabla) \mathbf{v}$ representa adecuadamente los efectos convectivos del fluido, es un término no lineal debido a que contiene productos de variables y con ello origina dificultades matemáticas en el análisis de los flujos.

Finalmente, la ecuación de movimiento para un elemento de volumen, en un fluido incompresible, viene dada por la suma de fuerzas involucradas por unidad de volumen, tales como: fuerza de presión $(-\nabla P)$, fuerza viscosa $(\nabla \cdot \tau)$, donde $\tau$ representa el tensor de esfuerzos viscosos, y la fuerza gravitacional $\rho \mathrm{g}$, igualada al producto de la masa por unidad de volumen y la aceleración

$$
\rho \frac{d \mathbf{v}}{d t}=-\nabla P+\nabla \cdot \tau+\rho \mathbf{g} ;
$$

para el caso de fluidos no viscosos, ideales, la fuerza $\nabla \cdot \tau$ no aparece.

Finalmente requerimos de la ecuación que exprese las relaciones termodinámicas del fluido. 


\subsection{La Ecuación de Energía}

La distribución de temperatura se rige por una ecuación que describe el balance de calor en cada elemento unitario de volumen promedio, la cual es una expresión de la primera ley de la termodinámica. Representando por $c_{p}$ el calor específico, la razón de cambio de calor contenido en este elemento de volumen está dado por $\rho c_{p} \frac{\partial T}{\partial t}$, el cual a su vez es el resultado de agregar (o quitar) calor en el transporte de calor advectivo por movimiento de fluidos intersticiales, por conducción térmica, y por producción (o absorción) de calor por reacciones químicas, si éstas se presentan.

Así, la entrada de calor advectivo (Landau y Lifschitz [16]) es

$$
-\left(\rho c_{p}\right) \nabla \cdot(T \mathbf{v})=-\left(\rho c_{p}\right)(\mathbf{v} \cdot \nabla T+T \nabla \cdot \mathbf{v})
$$

si el fluido es incompresible, entonces esta ecuación se reduce a

$$
-\left(\rho c_{p}\right) \nabla \cdot(T \mathbf{v})=-\left(\rho c_{p}\right) \mathbf{v} \cdot \nabla T
$$

Por otra parte, el flujo de calor puede ser expresado como $-\eta \nabla T$, donde $\eta$ es la conductividad térmica del medio. La razón de acumulación de calor por volumen unitario es entonces

$$
\nabla \cdot(\eta \nabla T)=\eta \nabla^{2} T
$$

esta simplificación se obtiene cuando el medio es localmente homogéneo $(\eta=c t e$.$) . Si además se presentan reacciones químicas y denotando por$ $Q$ la energía calorífica asociada a dichas reacciones, se tiene entonces que la ecuación de energía toma la forma

$$
\rho c_{p} \frac{\partial T}{\partial t}=-\left(\rho c_{p}\right) \mathbf{v} \cdot \nabla T+\eta \nabla^{2} T+Q
$$

reordenando (2.13) y dividiendo entre $\rho c_{p}$, tenemos

$$
\frac{\partial T}{\partial t}+\mathbf{v} \cdot \nabla T=\frac{1}{\rho c_{p}}\left(\eta \nabla^{2} T+Q\right)
$$

observamos que el lado izquierdo no es otra cosa que la derivada total de la temperatura, así que la ecuación de energía finalmente toma la forma

$$
\frac{d T}{d t}=\frac{1}{c_{p} \rho}\left(\eta \nabla^{2} T+Q\right) .
$$


De esta manera hemos expresado en forma breve las tres ecuaciones básicas para describir el flujo de un fluido.

Por lo tanto, el comportamiento de un fluido viscoso incompresible, sujeto a fuerzas de presión y gravitacionales y en donde no se presentan reacciones químicas, queda determinado matemáticamente por el siguiente sistema de ecuaciones

$$
\left.\begin{array}{l}
\nabla \cdot \mathbf{v}=0 \\
\rho \frac{d \mathbf{v}}{d t}=-\nabla P+\nabla \cdot \tau+\rho \mathbf{g} \\
\frac{d T}{d t}=\frac{\eta}{c_{p} \rho} \nabla^{2} T .
\end{array}\right\}
$$

Ahora, veamos cuales son las transformaciones que sufren estas ecuaciones cuando se trata con fluidos en medios porosos.

\subsection{Dinámica de Fluidos aplicada a Medios Porosos}

Podemos definir de manera intuitiva un medio poroso como un material sólido que contiene agujeros pequeños (poros), los cuales en general están interconectados; al espacio dentro del medio poroso que no es parte de la materia sólida se le llama espacio vacío (o espacio poroso). De aquí que las propiedades significativas de un medio poroso sean la porosidad, la cual es una medida del espacio poroso y por lo tanto de la capacidad de fluir del medio, y la permeabilidad, la cual indica la facilidad con la cual el fluido puede atravesar el medio bajo la acción de una presión.

El flujo de un fluido viscoso a través de un medio poroso es un caso especial del problema general del flujo de fluidos viscosos entre fronteras impermeables. Como los poros del medio son fijos y las superficies que los limitan se describen geométricamente, el flujo a través de estos poros está, en principio, sujeto a una descripción detallada por medio de las ecuaciones clásicas de la hidrodinámica. 
Sin embargo, cuando estudiamos el comportamiento de fluidos a través de medios porosos observamos que existen diferencias importantes con respecto

a la teoría clásica de flujos viscosos; estas diferencias se sitúan esencialmente en la expresión del sistema de ecuaciones dinámicas correspondientes. Ciertamente la ecuación de continuidad y las definiciones termodinámicas de un fluido deben conservarse en cualquier sistema hidrodinámico. Sin embargo, las reacciones dinámicas de un fluido que pasa a través de canales finos de un medio poroso, desde un punto de vista macroscópico, aparecen en formas bastante diferentes, estas diferencias se basan primordialmente en la Ley de Darcy, la cual termodinámicamente será complementada con la aproximación de Boussinesq.

\subsection{Ley de Darcy}

En el año de 1856 Henry Darcy se interesó en las características del flujo de fluidos a través de filtros de arena, para lo cual recurrió a un estudio experimental del problema, y fue de los primeros en establecer una base real de la teoría cuantitativa del flujo de fluidos homogéneos a través de medios porosos. Estos experimentos clásicos dieron el resultado conocido como Ley de Darcy, el cual establece que la velocidad de un fluido que fluye a través de un medio poroso es directamente proporcional al gradiente de presión que actúa sobre el fluido, expresada en términos vectoriales obtenemos

$$
\mathbf{v}=-\frac{k}{\mu} \nabla P,
$$

donde $\mu$ es la viscosidad del fluido. En estas ecuaciones la permeabilidad $k$ puede variar de un punto a otro, dependiendo de la influencia con la cual se manifiestan tanto la densidad como la viscosidad del fluido en el medio poroso y puede ser diferente para cada componente de la velocidad, lo cual nos llevaría a indicar la ley de Darcy en términos de las componentes de la velocidad e indicando la permeabilidad correspondiente para cada una de estas componentes. Sin embargo, si consideramos el medio como isotrópico, tomaremos entonces a $k$ como constante en cualquier dirección.

Sin embargo, esta ecuación aún no es suficientemente general, pues si una fuerza F, actúa sobre el fluido, ésta afectará, al igual que el gradiente de presión, a la velocidad, en tales casos la ley de Darcy toma la forma 


$$
\mathbf{v}=-\frac{k}{\mu}(\nabla P-\mathbf{F})
$$

Ahora bien, si la única fuerza externa $\mathrm{F}$ que actúa sobre el fluido es debida a la gravedad, la cual sólo tiene componente vertical, entonces la ecuación anterior para la velocidad, se puede plantear en términos de las componentes respectivas

$$
\left.\begin{array}{l}
v_{x}=-\frac{k}{\mu} \frac{\partial P}{\partial x} \\
v_{y}=-\frac{k}{\mu} \frac{\partial P}{\partial y} \\
v_{z}=-\frac{k}{\mu}\left(\frac{\partial P}{\partial z}+\rho g\right) .
\end{array}\right\}
$$

Sin embargo, aún estas ecuaciones para las componentes de la velocidad se verán todavía modificadas por la aproximación de Boussinesq.

\subsection{Aproximación de Boussinesq}

Como hemos indicado, en un medio poroso pueden existir fuerzas exteriores, generalmente producidas por el campo gravitacional de la tierra, y las diferencias de densidad debidas a gradientes de temperatura que pueden poner un fluido viscoso en movimiento, con lo cual el transporte de calor a través del medio se conoce con el nombre de conducción cuando el fluido darciano está estático ( $\sin$ movimiento), y convección cuando el fluido darciano está en movimiento. En particular, si existen gradientes de temperatura, esto producirá movimiento en el fluido debido a las diferencias de densidad que se generarán dentro del mismo, es entonces cuando decimos que la transferencia de calor se da por convección natural. Por lo tanto, la variación de la densidad con la temperatura representado por el coeficiente de expansión térmica $\beta$ es un factor importante en la convección natural.

Suponiendo que las diferencias de temperatura son pequeñas cuando describimos este movimiento, entonces será suficiente usar la aproximación de Boussinesq, la cual indica que las variaciones en la densidad $\rho$ son insignificantes excepto en el término de la fuerza externa (debida a la gravedad y llamada comúnmente fuerza de flotación) donde la variación inducida por la 
temperatura da origen a la fuerza $\mathbf{F}=\rho \mathbf{g}$ en (2.18). Como ejemplo, consideremos una situación inicial en la cual una capa de fluido estacionario frío (por lo tanto, más pesado) se encuentra encima de otra capa estacionaria más tibia (más ligera). Bajo ciertas condiciones, ésta puede ser una situación inestable, lo cual significa que incluso un disturbio pequeño puede dar como resultado una situación totalmente distinta, provocada por corrientes convectivas. La difusión molecular y la conducción térmica tienden a reducir estas corrientes suavizando hasta desaparecer las diferencias de densidad y estabilizando nuevamente el fluido.

Por lo tanto, la densidad $\rho$ en la fuerza de flotación satisface la siguiente ecuación de estado

$$
\rho=\rho_{o}\left(1-\beta\left(T-T_{o}\right)\right),
$$

(en donde el subíndice se refiere a un estado de referencia), la cual no es más que la correspondiente aproximación lineal de la ley de estado general $\rho=\rho(P, T)$. Así, la fuerza de flotación $\mathbf{F}$ por unidad de volumen toma entonces la forma

$$
\mathbf{F}=\rho_{o}\left(1-\beta\left(T-T_{o}\right)\right) \mathrm{g} .
$$

De esta manera, las ecuaciones para las componentes de la velocidad en un fluido darciano sujeto a la aproximación de Boussinesq son

$$
\left.\begin{array}{ll}
v_{x}=-\frac{k}{\mu} \frac{\partial P}{\partial x} & \\
v_{y}=-\frac{k}{\mu} \frac{\partial P}{\partial y} & \\
v_{z}=-\frac{k}{\mu}\left(\frac{\partial P}{\partial z}+\rho_{o} g\left(i-\beta\left(T-T_{o}\right)\right)\right), \quad \beta>0
\end{array}\right\}
$$

Finalmente, en el caso de convección natural en medios porosos para flujos incompresibles y viscosos sujetos a la aproximación de Boussinesq, el sistema de ecuaciones en forma vectorial que se obtiene es

$$
\left.\begin{array}{rl}
\nabla \cdot \mathbf{v} & =0 \\
\mathbf{v} & =-\frac{k}{\mu}\left(\nabla P+\rho_{o}\left(1-\beta\left(T-T_{0}\right)\right) \mathrm{g}\right) \\
\frac{d T}{d t} & =\frac{\eta}{c_{p} \rho} \nabla^{2} T .
\end{array}\right\}
$$




\subsection{Condiciones Iniciales y de Frontera}

Hemos descrito ya las ecuaciones que describen el flujo a través de un medio poroso; aunado a esto, debemos especificar las condiciones que deben satisfacer las ecuaciones en la frontera del dominio considerado. Si el problema es no estacionario, es decir, las variables también dependen del tiempo, entonces las condiciones de frontera deben especificarse para todo tiempo $t \geq 0$. De la misma manera deben indicarse las condiciones iniciales, esto es las condiciones que se satisfacen en todos los puntos del dominio en el instante de tiempo en que se inicia el proceso. Así, con esta información y para determinar matemáticamente un problema de flujo de fluidos requerimos:

a) definir la geometría de la frontera del problema;

b) determinar la variable (o variables) dependiente(s) por medio de la cual el flujo es descrito;

c) especificar las ecuaciones diferenciales parciales que deberán satisfacer las variables dependientes en todos los puntos dentro del dominio;

d) especificar las condiciones de frontera que deberán satisfacer las variables dependientes en todos los puntos de la frontera;

e) establecer las condiciones iniciales cuando el tiempo es una de las variables independientes.

Si denotamos la frontera por S y la variable dependiente por $\Phi$, las condiciones de frontera de mayor interés físico pueden tomar una de las siguientes formas:

a) $\Phi$ está definida sobre $\mathrm{S}$ (condición tipo Dirichlet).

b) $\frac{\partial \Phi}{\partial n}$ está definida sobre $\mathrm{S}$ (condición tipo Neumann).

c) $\frac{\partial \Phi}{\partial n}+h \Phi$ está definida sobre $\mathrm{S}(h$ puede ser función de $(x, y, z)$ ) (condición tipo Cauchy).

En nuestro caso, consideraremos sólo condiciones de frontera Dirichlet y mixtas (Dirichlet en una parte de la frontera y Neumann en otra). 


\section{Capítulo 3}

\section{Problema Continuo}

Resumen: Se expone el problema a resolver en forma continua, asimismo se realiza la simplificación de la ecuación de movimiento mediante la definición de la función de corriente, una vez hecho esto se procede a adimensionalizar el problema continuo.

\subsection{Introducción}

Consideremos una cavidad cuadrada, llena con un medio poroso, y dentro de la cual se encuentra un fluido viscoso e incompresible y supongamos que la aproximación de Boussinesq (las variaciones de densidad son insignificantes, excepto en el término de la fuerza de flotación) se satisface a través de todo el dominio, entonces las ecuaciones para las componentes de la velocidad en dos dimensiones vienen dadas por.

$$
\begin{aligned}
& u^{\prime}=-\frac{k}{\mu} \frac{\partial P}{\partial x^{\prime}} \\
& v^{\prime}=-\frac{k}{\mu}\left(\frac{\partial P}{\partial z^{\prime}}+\rho_{e} g\left(1-\beta\left(T-T_{o}\right)\right)\right)
\end{aligned}
$$

las cuales están acopladas a la ecuación de energía, dada por

$$
\rho c_{p}\left(\frac{\partial T}{\partial t^{\prime}}+u^{\prime} \frac{\partial T}{\partial x^{\prime}}+v^{\prime} \frac{\partial T}{\partial z^{\prime}}\right)=\eta \nabla^{2} T,
$$

donde 
$k$-permeabilidad del medio poroso $\left(\mathrm{m}^{2}\right)$.

$\beta$-coeficiente de expansión térmica $\left(1 /{ }^{\circ} \mathrm{K}\right)$.

$\mu$-coeficiente de viscosidad dinámica $(\mathrm{kg} / \mathrm{ms})$.

$\rho$-densidad $\left(\mathrm{kg} / \mathrm{m}^{3}\right)$.

$\rho_{e}$-densidad de referencia $\left(\mathrm{kg} / \mathrm{m}^{3}\right)$.

$\eta$-conductividad térmica del fluido $\left(m \mathrm{~kg} / \mathrm{s}^{3}{ }^{\circ} \mathrm{K}\right)$.

Estas y otras cantidades extras que aparecen en estas ecuaciones, así como en otras que obtendremos posteriormente, pueden consultarse en el apéndice A.

A continuación, procederemos a simplificar las ecuaciones de movimiento así como la de energía.

\subsection{Simplificación de la ecuación de movimien- to vía función de corriente}

La geometría del problema, así como las condiciones de frontera correspondientes, tanto para el problema original como para el problema adimensional respectivo, se indican en la figura 1.

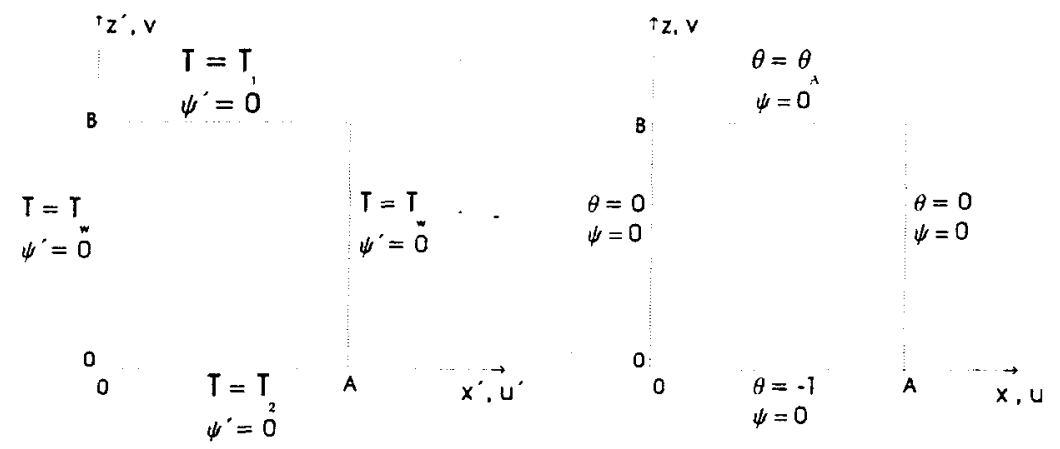

Figura 1. Geometría y nomenclatura para la cavidad del flujo

Considerando que el sistema a tratar tiene propiedades físicas constantes, las ecuaciones (3.1) se pueden combinar y transformar en una sola, mediante el concepto de función de corriente $\psi$, la cual se define a través de la velocidad del fluido como 


$$
\left.\begin{array}{l}
u^{\prime}=\frac{\partial \psi^{\prime}}{\partial z^{\prime}} \\
v^{\prime}=-\frac{\partial \psi^{\prime}}{\partial x^{\prime}}
\end{array}\right\}
$$

Usando estas relaciones, veremos cómo el término que contiene a la presión en el sistema (3.1) desaparece cuando calculamos el laplaciano de $\psi$.

Dado que

$$
\nabla^{2} \psi^{\prime}=\frac{\partial^{2} \psi^{\prime}}{\partial x^{\prime 2}}+\frac{\partial^{2} \psi^{\prime}}{\partial z^{\prime 2}}
$$

entonces por las ecuaciones (3.3) y (3.4), tenemos que

$$
\nabla^{2} \psi^{\prime}=\frac{k}{\mu} \frac{\partial^{2} P}{\partial x^{\prime} \partial z^{\prime}}-\frac{k g \beta \rho_{e}}{\mu} \frac{\partial T}{\partial x^{\prime}}-\frac{k}{\mu} \frac{\partial^{2} P}{\partial z^{\prime} \partial x^{\prime}},
$$

considerando que $\frac{\partial^{2} P}{\partial x^{\prime} \partial z^{\prime}}=\frac{\partial^{2} P}{\partial z^{\prime} \partial x^{\prime}}$, se tiene que los términos que contienen a la presión desaparecen, y entonces la ecuación (3.5) se reduce a

$$
\nabla^{2} \psi^{\prime}=-\frac{k g \beta \rho_{e}}{\mu} \frac{\partial T}{\partial x^{\prime}} .
$$

Además la condición de incompresibilidad del fluido

$$
\nabla \cdot\left(u^{\prime}, v^{\prime}\right)=0
$$

se satisface automáticamente debido a la forma en que se definen $u^{\prime}$ y $v^{\prime}$.

Así, el sistema (3.1)-(3.2) está dado por

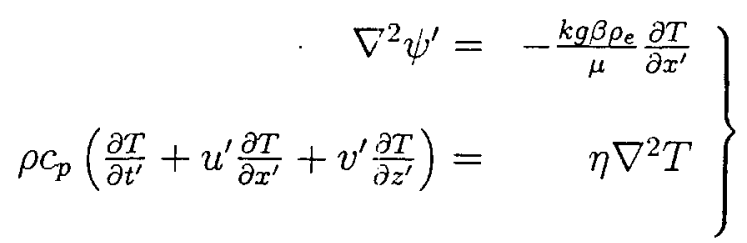

sujeto a las condiciones de frontera (véase figura 1).

$$
\begin{aligned}
& T=T_{w} \text { en } x^{\prime}=0 \text { y } x^{\prime}=A \\
& T=T_{1} \text { en } z^{\prime}=0 \\
& T=T_{2} \text { en } z^{\prime}=B, \\
& \psi^{\prime}=0 \text { en } x^{\prime}=0 \text { y } x^{\prime}=A \\
& \psi^{\prime}=0 \text { en } z^{\prime}=0 \text { y } z^{\prime}=B
\end{aligned}
$$


La condición de frontera $\psi^{\prime}=0$ corresponde a la condición de deslizamiento nulo del fluido viscoso en las paredes de la cavidad que lo contiene.

\subsection{Problema Adimensional}

Procederemos ahora a adimensionalizar estas ecuaciones definiendo nuevas variables a partir de referencias conocidas

$$
\begin{gathered}
x=\frac{x^{\prime}}{B} \\
z=\frac{z^{\prime}}{B} \\
L=\frac{A}{B} \\
R=\frac{B k g \beta \rho_{e}}{\kappa \mu}\left(T_{w}-T_{2}\right) \\
t=\frac{t^{\prime} \kappa}{B^{2}} \\
\psi=\frac{\psi^{\prime}}{\kappa} \\
\theta=\frac{T}{T_{i w}-T_{w}}
\end{gathered}
$$

En particular consideraremos que $A=B$, con lo que $L=1$. Sustituyendo estas nuevas variables en la primera ecuación del sistema (3.8), tenemos:

$$
\frac{\kappa}{B^{2}} \nabla^{2} \dot{\psi}=-\frac{k g \beta \rho_{e}}{\mu} \frac{\left(T_{w}-T_{2}\right)}{B} \frac{\partial \theta}{\partial x}
$$

es decir

$$
\nabla^{2} \psi=-\frac{B k g 3 \rho_{e}}{\kappa \mu}\left(T_{w}-T_{2}\right) \frac{\partial \theta}{\partial x},
$$

finalmente la ecuación adimensional çue se obtiene es

$$
\nabla^{2} v=-R \frac{\partial \theta}{\partial x}
$$


$\operatorname{con}$

$$
R=\frac{B k g \beta \rho_{e}}{\kappa \mu}\left(T_{w}-T_{2}\right)
$$

El parámetro $\mathrm{R}$ es el número de Darcy-Raleigh, el cual hemos mencionado anteriormente, que representa el balance entre las fuerzas de flotación y los procesos difusivos que retardan el movimiento del fluido y tienden a estabilizarlo.

Procedemos de manera análoga para la segunda ecuación (ecuación de energía) del sistema (3.8)

$$
\frac{\rho c_{p} \kappa\left[T_{w}-T_{2}\right]}{B^{2}}\left(\frac{\partial \theta}{\partial t}+u \frac{\partial \theta}{\partial x}+v \frac{\partial \theta}{\partial z}\right)=\frac{\eta\left[T_{w}-T_{2}\right]}{B^{2}} \nabla^{2} \theta
$$

además, considerando que la conductividad térmica $\eta$ está definida como

$$
\eta=\rho c_{p} \kappa,
$$

encontramos finalmente que la ecuación adimensional para la energía es

$$
\frac{\partial \theta}{\partial t}+u \frac{\partial \theta}{\partial x}+v \frac{\partial \theta}{\partial z}=\nabla^{2} \theta
$$

Por lo tanto, el problema original lo podemos transformar en un problema adimensional con $t>0$, confinado a una cavidad cuadrada unitaria $\Omega=$ $(0,1) \times(0,1)$, cuya frontera denotaremos por $\Gamma$; en general, consideraremos el dominio espacial-temporal como $Q=\Omega \times(0, T)$ en donde se satisface el siguiente sistema de ecuaciones

$$
\left.\begin{array}{rl}
-\frac{1}{R} \nabla^{2} \psi & =\frac{\partial \theta}{\partial x} \\
\frac{\partial \theta}{\partial t}-\nabla^{2} \theta+\mathrm{v} \cdot \nabla \theta & =0
\end{array}\right\}
$$

donde

$$
\mathbf{v}=(u, v)=\left(\frac{\partial \psi}{\partial z},-\frac{\partial \psi}{\partial x}\right)
$$


Por último, para que el problema esté bien planteado, consideramos tanto para $\theta$ como para $\psi$ condiciones iniciales y de frontera, éstas últimas son:

a) Para la primera parte del trabajo, tipo Dirichlet, tanto $\psi$ como para $\theta$, para todo $t>0$.

$$
\begin{aligned}
& \theta=0 \text { en } x=0 \quad y \quad x=1 \\
& \theta=-1 \text { en } z=0 \\
& \theta=\theta_{A} \text { en } z=1 \text {, } \\
& \psi=0 \text { en } x=0 \text { y } \quad x=1 \\
& \psi=0 \quad \text { en } \quad z=0 \quad y \quad z=1,
\end{aligned}
$$

en donde $\theta_{A}$ es un valor arbitrario para la temperatura que nosotros fijamos.

b) Para la segunda parte del trabajo, serán tipo Dirichlet para $\psi$ y mixtas para $\theta$, y serán indicadas en su momento.

Para ambos casos la condición inicial es

$$
\theta(x, z, t=0)=0, \quad \psi(x, z, t=0)=0 \quad \text { en } \Omega .
$$

Entonces, el problema a resolver consiste en encontrar

$$
\psi(x, z, t) \text { y } \theta(x, z, t) . \quad \text { con } \quad(x, z, t) \in Q,
$$

que sean soluciones simultáneas de (3.14) sujetas a las condiciones de frontera e iniciales respectivas. 


\section{Capítulo 4}

\section{Problema Discreto}

Resumen: Se procede a la discretización del problema, en primer lugar se realiza la discretización temporal a través del método de Gear modificado, con lo cual se obtienen ecuaciones diferenciales no lineales y estacionarias en cada nivel de tiempo, posteriormente presentamos el esquema numérico para resolver estas ecuaciones, las cuales al ser discretizadas en el espacio dan lugar a un par de sistemas de ecuaciones lineales en cada nivel de tiempo. Finalizamos describiendo el número de Nusselt correspondiente para cada una de las fronteras de la cavidad.

\subsection{Introducción}

Para resolver el sistema de ecuaciones diferenciales (3.14), obtenido en el problema continuo, se nos presentan básicamente las siguientes dificultades :

1) Las ecuaciones están acopladas, es decir, debemos resolver simultáneamente para $\theta$ y para $\psi$.

2) El sistema es dependiente del tiempo y no lineal porque la segunda ecuación de (3.14) lo es, pues $\psi$ es incógnita, y por lo tanto $u$ y $v$ también lo son.

Para encontrar la solución numérica, debemos proceder a realizar la discretización de cada una de las ecuaciones diferenciales parciales. 
En primer lugar, discretizamos las ecuaciones respecto al tiempo, con lo cual obtenemos un sistema de ecuaciones diferenciales parciales estacionarias no lineales en cada nivel de tiempo. Este sistema lo resolvemos mediante un método iterativo de punto fijo, el cual nos conduce en cada nivel de tiempo a resolver dos sistemas de ecuaciones lineales desacoplados. La discretización espacial de este sistema puede realizarse mediante elemento finito (estableciendo previamente una formulación variacional) o mediante diferencias finitas; en cualquier caso se obtiene un sistema de ecuaciones algebraicas lineales. Nuestros resultados se obtienen mediante esquemas de diferencias finitas de segundo orden.

\subsection{Problema Semidiscreto (Discretización en Tiempo)}

Recordemos el sistema a resolver

$$
\left.\begin{array}{rl}
-\frac{1}{R} \nabla^{2} \psi & =\frac{\partial \theta}{\partial x} \\
\frac{\partial \theta}{\partial t}-\nabla^{2} \theta+\mathbf{v} \cdot \nabla \theta & =0
\end{array}\right\}
$$

sujeto a ciertas condiciones de frontera e iniciales.

En la ecuación de energía aproximamos la primera derivada parcial respecto al tiempo mediante el esquema de Gear modificado, el cual es de segundo orden e incondicionalmente estable, es decir

$$
\frac{\partial \theta}{\partial t}(x, z,(n+1) \Delta t) \approx \frac{\frac{3}{2} \theta^{n+1}-2 \theta^{n}+\frac{1}{2} \theta^{n-1}}{\Delta t} ; \quad n \geq 1
$$

donde

$$
\theta^{k}(x, z, t) \approx \theta(x, z, k \Delta t) .
$$

De esta forma, nuestro sistema de ecuaciones discretizado en el tiempo está dado como

$$
\left.\begin{array}{rl}
\alpha \theta^{n+1}-\nabla^{2} \theta^{n+1}+\left(u^{n+1}, v^{n+1}\right) \cdot \nabla \theta^{n+1} & =f^{n+1} \\
-\frac{1}{R} \nabla^{2} \psi^{n+1} & =\left(\frac{\partial \theta}{\partial x}\right)^{n+1},
\end{array}\right\}
$$


en donde

$$
\alpha=\frac{3}{2 \Delta t}, y f^{n+1}=\frac{2 \theta^{n}-\frac{1}{\theta^{n-1}}}{\Delta t},
$$

más las condiciones de frontera dadas por (3.15).

Por lo que para resolver este par de ecuaciones diferenciales parciales, debemos encontrar $\psi(x, z,(n+1) \Delta t)$ y $\theta(x, z,(n+1) \Delta t)$ que sean solución de (4.2) para cada tiempo $(n+1) \Delta t$. Entonces si renombramos

$$
\begin{aligned}
& \theta=\theta^{n+1} \\
& \psi=\psi^{n+1}
\end{aligned}
$$

en cada nivel de tiempo, observamos que debemos resolver un sistema estacionario no-lineal de ecuaciones diferenciales parciales de la forma

$$
\left.\begin{array}{rl}
-\frac{1}{R} \nabla^{2} \psi & =\frac{\partial \theta}{\partial x} \\
\alpha \theta-\nabla^{2} \theta+(u, v) \cdot \nabla \theta & =f
\end{array}\right\}
$$

más las condiciones de frontera (3.15).

La manera en que resolveremos este sistema en cada nivel $(n+1) \Delta t$ de tiempo, es haciendo uso de un método iterativo de punto fijo, mismo que explicaremos en la siguiente sección.

\subsection{Método de Solución para el Problema Es- tacionario}

El esquema para resolver este sistema, es una variante de otros métodos, que han mostrado ser eficientes para flujos más complicados respecto a medios no porosos, relacionados con las ecuaciones de Navier-Stokes así como a la aproximación de Boussinesq [10].

Respecto a la ecuación de energía consideraremos

$$
\Upsilon(\theta, \dot{\psi}) \equiv \alpha \theta-\nabla^{2} \theta+(u, v) \cdot \nabla \theta-f .
$$

Así el sistema (4.3) es equivalente a

$$
\left.\begin{array}{rl}
\Upsilon\left(\theta, \iota^{\prime}\right) & =0 \\
-\frac{1}{R} \nabla^{2} \iota & =\frac{\partial \theta}{\partial x},
\end{array}\right\}
$$

más las condiciones de frontera (3.15). 
Considerando que el sistema de ecuaciones a resolver consiste de dos ecuaciones elípticas, una de ellas lineal y la otra no-lineal. En lo que sigue haremos uso del siguiente método iterativo de punto fijo.

Dados

$$
\begin{aligned}
\theta^{0} & =2 \theta^{n}-\theta^{n-1} \\
\psi^{0} & =2 \psi^{n}-\psi^{n-1},
\end{aligned}
$$

interpolaciones lineales de los valores conocidos en los tiempos anteriores, resolvemos el siguiente sistema hasta obtener convergencia en $\theta$

$$
\left.\begin{array}{ll}
\theta^{m+1}= & \theta^{m}-\lambda\left(\alpha I-\nabla^{2}\right)^{-1} \Upsilon\left(\theta^{m}, \psi^{m}\right) \\
\theta^{m+1}= & \theta^{n} \quad \text { en } \Gamma \\
-\frac{1}{R} \nabla^{2} \psi^{m+1}= & \frac{\partial \theta^{m+1}}{\partial x} \quad \text { en } \Omega \\
\psi^{m+1}= & \text { en } \Gamma,
\end{array}\right\}
$$

en donde

$$
0<\lambda<1 \text { en } \Omega
$$

y el superíndice $m$ indica las iteraciones del método de punto fijo a considerar.

Finalmente tomamos

$$
\theta^{n+1}=\theta^{m+1} \quad y \quad \psi^{n+1}=\psi^{m+1} .
$$

Observemos que la convergencia en $\theta$, implica la convergencia de $\psi$, obteniendo así la convergencia a la solución $\left(\theta^{n+1}, \psi^{n+1}\right)$ del sistema.

Es importante hacer notar, que la primera ecuación del sistema (4.6) podemos sustituirla por la ecuación equivalente

$$
\left(\alpha I-\nabla^{2}\right) \theta^{m+1}=\left(\alpha I-\nabla^{2}\right) \theta^{m}-\lambda \Upsilon\left(\theta^{m}, \psi^{m}\right) .
$$

De esta manera en cada iteración tenemos que resolver dos problemas elípticos lineales simétricos no-acoplados. La parte no simétrica en la ecuación $(4.4),(u, v) \cdot \nabla \theta$, queda en el lado derecho como una consecuencia del método iterativo.

Para iniciar este método debemos contar con dos valores iniciales para $\theta$ y dos para $\psi$, es decir cuando $n=1$ los valores que obtendremos, una vez que aplicamos el método de punto fijo, son los correspondientes al segundo nivel de tiempo $\theta^{2}, \psi^{2}$, razón por lo cual necesitamos los valores respectivos de los dos primeros niveles, esto es debemos conocer los valores de $\theta^{0}, \theta^{1}, \psi^{0}, \psi^{1}$.

Como sólo conocemos un valor inicial para $\theta$ y uno para $\psi\left(\begin{array}{llll}\theta^{0} & y & \psi^{0}\end{array}\right.$ son conocidos), la manera de obtener $\theta^{1}$ y $u^{1}$ es aproximar la derivada temporal de la primera ecuación de (4.5) por medio del esquema de Euler, y 
aplicar entonces el mismo método de punto fijo para encontrar los valores correspondientes al primer nivel de tiempo.

El esquema de Euler para aproximar $\frac{\partial \theta}{\partial t}$ viene dado por

$$
\frac{\partial \theta}{\partial t}(n \Delta t) \approx \frac{\theta^{n+1}-\theta^{n}}{\Delta t} ; \quad n \geq 0
$$

Con lo que las ecuaciones resultantes tienen exactamente la misma forma que las del sistema (4.3), sólo que ahora :

$$
\alpha=\frac{1}{\Delta t}, \quad y \quad f=\frac{\theta^{n}}{\Delta t},
$$

Cabe aclarar que éste esquema es de primer orden, y puesto que nuestro esquema general a partir de la segunda iteración es de segundo orden tratamos de solventar este problema dividiendo $\Delta t$ en 5 subintervalos e iterando en cada uno de ellos hasta llegar a la convergencia y al finalizar el último subintervalo temporal tomamos los valores obtenidos para $\theta$ y para $\psi$ en ese nivel de tiempo como $\theta^{1}$ y $\psi^{1}$, respectivamente.

De esta manera, encontramos los valores para $\theta^{1}$ y $\psi^{1}$, y podemos continuar con el método iterativo para los siguientes tiempos usando el esquema de Gear modificado.

\subsection{Discretización en el espacio}

Como ya se mencionó antes, utilizamos esquemas de diferencias finitas de segundo orden para aproximar los términos convectivo y difusivo de $\theta$, esto es

$$
\begin{aligned}
& \frac{\partial \theta}{\partial x}\left(x_{i}, y_{j}\right) \quad \approx \frac{\theta_{i+1, j}-\theta_{i-1, j}}{2 h} \\
& \frac{\partial \theta}{\partial y}\left(x_{i}, y_{j}\right) \quad \approx \frac{\theta_{i, j+1}-\theta_{i, j-1}}{2 h} \\
& \frac{\partial^{2} \theta}{\partial x^{2}}\left(x_{i}, y_{j}\right) \quad \approx \frac{\theta_{i+1, j}-2 \theta_{i, j}+\theta_{i-1, j}}{h^{2}} \\
& \frac{\partial^{2} \theta}{\partial y^{2}}\left(x_{i}, y_{j}\right) \quad \approx \frac{\theta_{i, j+1}-2 \theta_{i, j}+\theta_{i, j-1}}{h^{2}}
\end{aligned}
$$

donde

$$
\begin{aligned}
& h=\frac{1}{N+1} \quad \text { con } N>0 \text { entero, } \\
& \mathrm{i}, \mathrm{j}=1, . ., \mathrm{N}, \text { y además, }
\end{aligned}
$$


$x_{i}=i * h$, y $y_{j}=j * h$,

$\theta_{i, j} \approx \theta\left(x_{i}, y_{j}\right)$.

Recordemos que estamos en el nivel de tiempo $(n+1) \Delta t$, por lo que en realidad tenemos

$$
\theta_{i, j}=\theta_{i, j,(n+1) \Delta t} \approx \theta\left(x_{i}, y_{j},(n+1) \Delta t\right)
$$

El proceso es análogo para $\frac{\partial v}{\partial x}, \frac{\partial v}{\partial y}, \frac{\partial^{2} \psi}{\partial x^{2}}$ y $\frac{\partial^{2} \psi}{\partial y^{2}}$.

De esta manera en el sistema (4.6), la relación para $\Upsilon$ discretizada en el espacio toma la forma

$$
\Upsilon(\Theta, \Psi)=(\alpha I+A) \Theta+\left(u_{i, j}, v_{i, j}\right) \cdot \nabla \Theta_{i, j}-F
$$

donde

I denota a la matriz identidad de orden $\mathrm{N}^{2} \times N^{2}$,

A es una matriz por bloques, de orden $\mathrm{N}^{2} \times N^{2}$, la cual proviene del laplaciano de $\theta$ y tiene la siguiente estructura

$$
\mathrm{A}=\frac{1}{h^{2}}\left(\begin{array}{ccccc}
\mathrm{A}_{1} & -\mathrm{I}_{1} & \mathrm{O}_{1} & \cdots & \mathrm{O}_{1} \\
-\mathrm{I}_{1} & \ddots & \ddots & \ddots & \vdots \\
\mathrm{O}_{1} & \ddots & \ddots & \ddots & \mathrm{O}_{1} \\
\vdots & \ddots & \ddots & \ddots & -\mathrm{I}_{1} \\
\mathrm{O}_{1} & \cdots & \mathrm{O}_{1} & -\mathrm{I}_{1} & \mathrm{~A}_{1}
\end{array}\right)
$$

y cada uno de los bloques son matrices de orden $\mathrm{N} \times N$ las cuales tienen la siguiente forma

$$
\mathbf{A}_{1}=\left(\begin{array}{ccccc}
4 & -1 & 0 & \cdots & 0 \\
-1 & \ddots & \ddots & \ddots & \vdots \\
0 & \ddots & \ddots & \ddots & 0 \\
\vdots & \ddots & \ddots & \ddots & -1 \\
0 & \cdots & 0 & -1 & 4,
\end{array}\right)
$$

$\mathrm{I}_{1}=$ matriz identidad de orden $N \times N$,

$\mathrm{O}_{1}=$ matriz nula de orden $N \times N$.

Además, tanto $\mathrm{A}$ como $\mathrm{A}_{1}$ son matrices simétricas y definidas positivas. 
Por otro lado, por (3.3)

$$
\begin{aligned}
\left(u_{i, j}, v_{i, j}\right) & =(u, v)\left(x_{i}, y_{j}\right)=\left(\frac{\partial \psi}{\partial y}\left(x_{i}, y_{j}\right),-\frac{\partial \psi}{\partial x}\left(x_{i}, y_{j}\right)\right) \\
\nabla \theta_{i, j} & =\nabla \theta\left(x_{i}, y_{j}\right),
\end{aligned}
$$

tenemos

$$
\begin{gathered}
\left(u_{i, j}, v_{i, j}\right) \cdot \nabla \theta_{i, j} \approx \\
\frac{\left(\psi_{i, j+1}-\psi_{i, j-1}\right)\left(\theta_{i+1, j}-\theta_{i-1, j}\right)-\left(\psi_{i+1, j}-\psi_{i-1, j}\right)\left(\theta_{i, j+1}-\theta_{i, j-1}\right)}{4 h^{2}},
\end{gathered}
$$

$\operatorname{con} i, j=1, \ldots, N$.

De esta manera el sistema de ecuaciones (4.6) toma finalmente la forma

$$
\left.\begin{array}{ccc}
(\alpha I+A) \Theta^{m+1}= & (\alpha I+A) \Theta^{m}-\lambda \Upsilon\left(\Theta^{m}, \Psi^{m}\right), & \lambda>0 \\
\frac{1}{R} A \Psi^{m+1}= & \left\{\frac{\theta_{i+1, j}^{m+1}-\theta_{i-1 . j}^{m+1}}{2 h}\right\}_{i, j=1}^{N} . &
\end{array}\right\}
$$

Así, hemos discretizado totalmente el problema y podemos aplicar un método adecuado para resolver los dos sistemas de ecuaciones algebráicas lineales desacoplados que se obtienen; para condiciones de frontera de tipo Dirichlet, tanto para $\theta$ como para $\psi$, los resolvemos haciendo uso del método iterativo SOR por bloques (apéndice B), mientras que cuando se tienen condiciones de frontera Dirichlet para $\psi$ y Mixtas para $\theta$ aplicamos el paquete de FISHPACK (apéndice C).

\subsection{Número de Nusselt}

Finalmente, una variable adimensional extra e importante que proporciona una forma alternativa del coeficiente de transferencia de calor ( el flujo de calor por unidad de área $\mathrm{q}$ es proporcional al gradiente de temperatura $\nabla \mathrm{T}$, $\mathrm{q}=-\mathrm{k} \nabla \mathrm{T}$, donde $\mathrm{k}$ es la conductividad térmica del fluido), es el número de Nusselt. Este número provee una medida del gradiente de temperatura en una superficie al gradiente de temperatura a través de un fluido.

Para calcular el número de Nusselt local correspondiente a cada una de las superficies que componen la frontera, usamos las soluciones correspondientes obtenidas de las ecuaciones que modelan el flujo. De esta manera, el número de Nusselt a lo largo de la frontera superior está definido como 


$$
N u=\left.\frac{L}{\theta_{A}-\bar{\theta}} \frac{\partial \theta}{\partial z}\right|_{z=1} .
$$

Mientras que para la superficie inferior, el número local de Nusselt se define como

$$
N u=\left.\frac{-L}{-1-\bar{\theta}} \frac{\partial \theta}{\partial z}\right|_{z=0} .
$$

$\mathrm{Y}$ a lo largo de la frontera lateral se tiene que el número local de Nusselt es

$$
N u=\left.\frac{1}{\bar{\theta}} \frac{\partial \theta}{\partial x}\right|_{x=0},
$$

en este caso sólo calculamos el número de Nusselt para la frontera lateral correspondiente a $\mathrm{x}=0$, ya que los mismos resultados se obtienen cuando $\mathrm{x}=1$.

Las derivadas que aparecen en el número de Nusselt se obtuvieron usando un esquema de diferencias finitas centradas, de segundo orden, con base en los resultados obtenidos de cada caso. 


\section{Capítulo 5}

\section{Resultados Numéricos}

Resumen: Mostramos las gráficas de la función corriente y de la temperatura, para diferentes condiciones de frontera, correspondientes a los resultados obtenidos en cada uno de los casos. En algunos de éstos, se muestran también las gráficas de los números de Nusselt promedio así como locales correspondientes a cada una de las fronteras de la cavidad.

\subsection{Introducción}

$\mathrm{Al}$ resolver los sistemas lineales, obtenidos de la discretización del problema original, observamos que las matrices que provienen del laplaciano conservan una estructura de bloques durante todo el proceso de discretización sin alterar las propiedades mencionadas en la sección anterior (simétrica, definida positiva, etc.); por ésta razón se eligió el método iterativo SOR por bloques (apéndice B) para encontrar la solución numérica del problema, y este método nos condujo finalmente a resolver $\mathrm{N}$ subsistemas lineales para cada uno de los dos sistemas lineales, los cuales a su vez los resolvimos haciendo uso del método de Cholesky.

Como todos estos últimos subsistemas conservan la misma matriz, independientemente del tiempo y de las iteraciones, esto nos da una ventaja computacional importante pues la factorización, que es una parte costosa del programa, la realizamos únicamente una vez, con lo que en cada nivel de tiempo sólo tenemos que realizar la sustitución hacia atrás y hacia adelante para cada iteración. 
Por otra parte, una característica muy importante del sistema de ecuaciones diferenciales es que, debido a la geometría del dominio y a las condiciones de frontera dadas tanto para $\theta$ como para $\psi$, esperamos obtener simetría en la gráfica de $\theta$ y antisimetría en la gráfica de $\psi$ alrededor de la línea $x=\frac{1}{2}$, en términos matemáticos

$$
\begin{array}{ll}
\theta(x, z, t)=\theta(1-x, z, t) & 0 \leq x \leq 1 \\
\psi(x, z, t)=-\psi(1-x, z, t) & 0 \leq x \leq 1,
\end{array}
$$

de donde se desprende que

$$
\begin{array}{cc}
\psi=0 & \text { en } x=\frac{1}{2} \\
\frac{\partial \theta}{\partial x}=0 & \text { en } x=\frac{1}{2} \\
\frac{\partial \psi}{\partial z}=0 & \text { en } x=\frac{1}{2},
\end{array}
$$

y puesto que la primera derivada de la, función corriente en la dirección de $\mathrm{z}$ es igual a cero para todo punto de la forma $\left(\frac{1}{2}, z\right)$ con $0 \leq z \leq 1$, entonces podemos concluir que, la componente horizontal de la velocidad $u$ es cero; por su parte, la componente vertical de la velocidad $v$ no necesariamente es cero en esos puntos.

Con estas consideraciones presentamos a continuación resultados numéricos, en primer lugar cuando las condiciones de frontera son de tipo Dirichlet para $\theta$ y para $\psi$, los cuales concuerdan en buena parte con los resultados obtenidos por Christopher [7] con un esquema de diferencias finitas de cuarto orden, posteriormente continuamos con los resultados numéricos obtenidos cuando tenemos condiciones de frontera Mixtas para $\theta$ y de tipo Dirichlet para $\psi$.

Para ambos casos, tomamos como valor para el parámetro $\lambda$ del método iterativo de punto fijo el valor $\lambda=0.9$ y criterio de convergencia (tolerancia) de $10^{-9}$.

\subsection{Condiciones de Frontera Dirichlet}

En todos los casos tenemos las siguientes condiciones de frontera: 


$$
\begin{aligned}
& \theta=-1 \text { en } z=0 \\
& \theta=0 \text { en } x=0 \quad y, x=1 \\
& \theta=\theta_{A} \text { en } z=1 \\
& \psi=0 \text { en } \Gamma
\end{aligned}
$$

Donde $\theta_{A}$ denota el valor en la frontera superior del dominio, el cual precisaremos en cada caso, así como otros parámetros necesarios.

Caso I) $\theta_{A}=-0.5, \quad \therefore=1,000, \quad h=\frac{1}{40}, \quad \Delta t=.0002$

Las paredes de la cavidad se encuentran a mayor temperatura que las fronteras inferior y superior de la cavidad por lo que el calentamiento ocurre a través de dichas fronteras laterales. Observese que inicialmente el fluido también se encuentra a mayor temperatura que la tapa y la base de la cavidad.
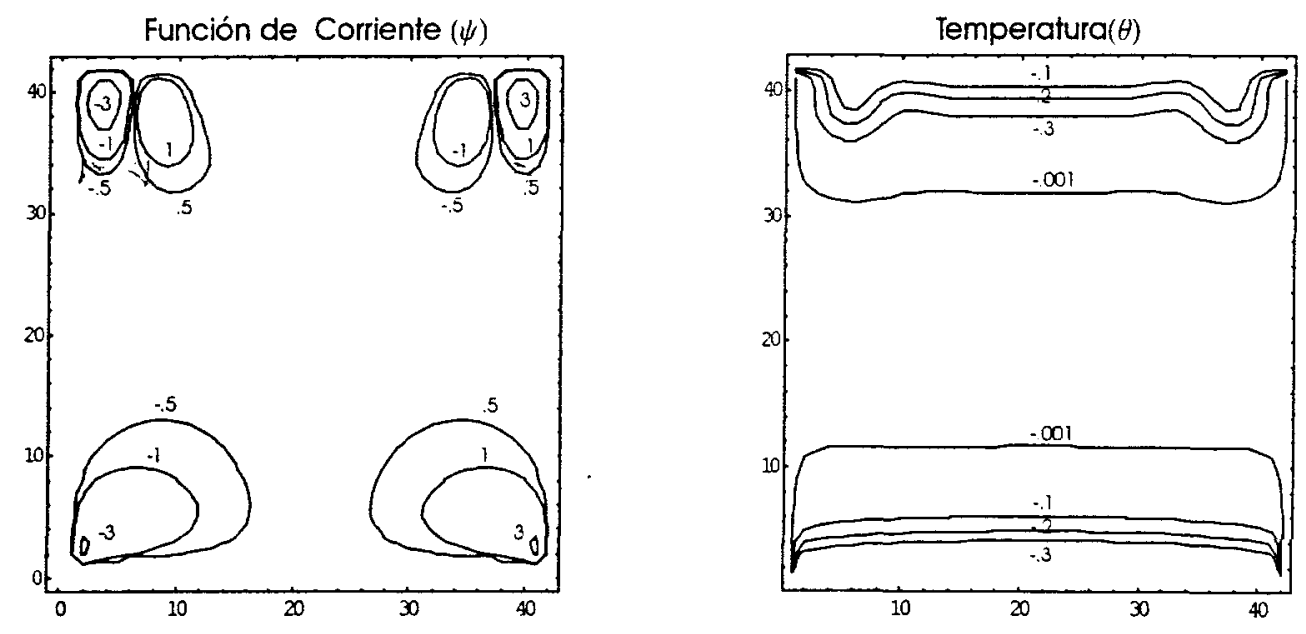

Figura 2

Contornos de la función de corriente y de la temperatura para $t=0.003$

En la figura 2 mostramos los contornos de la función corriente y de la temperatura, respectivamente, cuando comienza a desarrollarse el flujo.

El fluido se enfría en las superficies superior e inferior y se calienta en las paredes. Como observamos de la gráfica de la función corriente, el flujo 
comienza a desarrollarse en las esquinas. La circulación del fluido, en la parte superior, comienza como pequeñas burbujas de fluido frío que caen, alejándose de la frontera superior, hacia el cuerpo principal del mismo, el cual se encuentra a mayor temperatura; este enfriamiento y calentamiento provoca que la circulación se produzca en sentido negativo (en el sentido de las manecillas del reloj) en la esquina superior izquierda y, debido a la antisimetría de la función corriente, en el sentido positivo (en el sentido contrario a las manecillas del reloj) en la esquina superior derecha, a medida que el fluido que es calentado asciende cerca de la pared; muy cerca de estas burbujas se forma otro movimiento en contrasentido, respectivamente a cada una de ellas, a medida que el fluido que ha sido enfriado por la frontera superior desciende, alejándose de ésta. Al mismo tiempo, en la frontera inferior, se producen burbujas que giran en sentido negativo en la esquina izquierda y positivo en la esquina derecha.
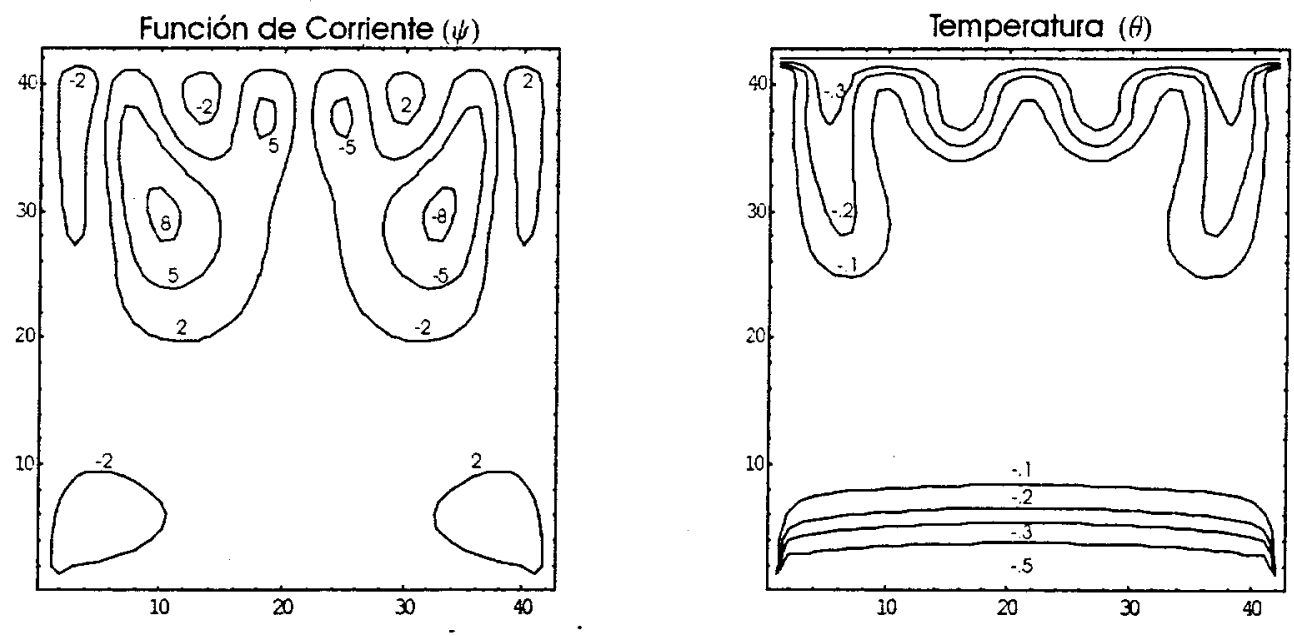

Figura 3

Contornos de la función de corriente y de la temperatura para $t=0.008$

La circulación es más lenta en los vórtices de las esquinas inferiores que en los de las esquinas superiores, lo cual se debe a que el fluido más frío y denso se encuentra debajo del fluido más caliente, con lo cual se inhibe el flujo, mientras que en los vórtices de la esquina superior, el fluido más frío está sobre el fluido tibio, lo cual incrementa la circulación.

Debido al calor que ingresa desde las paredes, los vórtices en las esquinas inferiores se extienden hacia arriba abarcando una mayor parte de la cavidad 
que aquella correspondiente a la de los vórtices de las esquinas superiores pues su crecimiento se ve limitado por la frontera superior.

De igual manera la gráfica para la temperatura muestra el hecho de que el fluido comienza a enfriarse cerca de las fronteras superior e inferior mientras conserva una temperatura más cálida cerca de las paredes y en el centro de la cavidad.

Al comparar este resultado con el correspondiente de Christopher observamos diferencias notables, las cuales básicamente se relacionan con el tiempo en que se alcanzan los mismos gráficos. Cuando $t=0.003$ obtenemos una distribución para la función corriente y la temperatura similar a la correspondiente de Christopher cuando $t=0.004$, estos resultados son los que presentamos en la figura 2 .
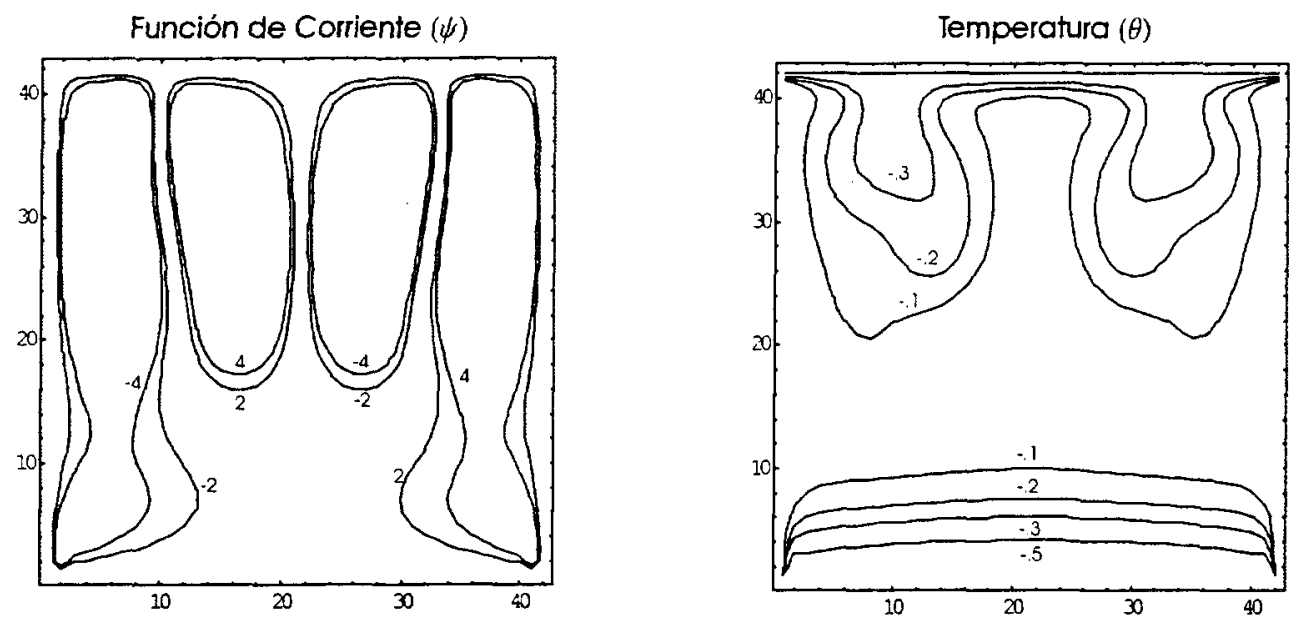

Figura 4

Contornos de la función de corriente y de la temperatura para $t=0.016$

Es importante aclarar que mientras nosotros presentamos los resultados obtenidos en la cavidad completa, Christopher, considerando el hecho de la simetría para la temperatura y la antisimetría para la funciń corriente, sólo presenta los resultados correspondientes a la mitad izquierda de la cavidad, esto es, cuando $0 \leq x \leq \frac{1}{2}$.

En la figura 3, la gráfica de la función corriente muestra que se ha formado otro par de vórtices adicionales a los anteriores, los cuales han continuado desarrollándose. Las celdas superiores que se formaron inicialmente han crecido, alargándose y cayendo hacia el centro de la cavidad donde se 
encuentra el fluido menos frío; mientras que los vórtices inferiores también crecen, aunque de manera más lenta.

La distribución de temperatura muestra que el fluido tibio que se encuentra cerca de las paredes y en el centro de la cavidad tiende a elevarse, reemplazando de esta forma al fluido frio que surge de la frontera superior.

En un tiempo posterior, al continuar desarrollándose el flujo, figura 4, los vórtices provenientes de las esquinas superior e inferior izquierdos se colapsan en una celda a lo largo de la pared, teniendo su contraparte, debido a que $\psi$ es antisimétrica, en la mitad derecha. Estas celdas continúan desarrollándose y creciendo con lo cual empujan a las celdas restantes hacia el centro de la cavidad. La gráfica correspondiente de la temperatura indica que el fluido más frío proveniente de la superficie superior sustituye al fluido tibio que se encuentra en el centro de la cavidad, ccupando ahora una mayor porción de la misma.
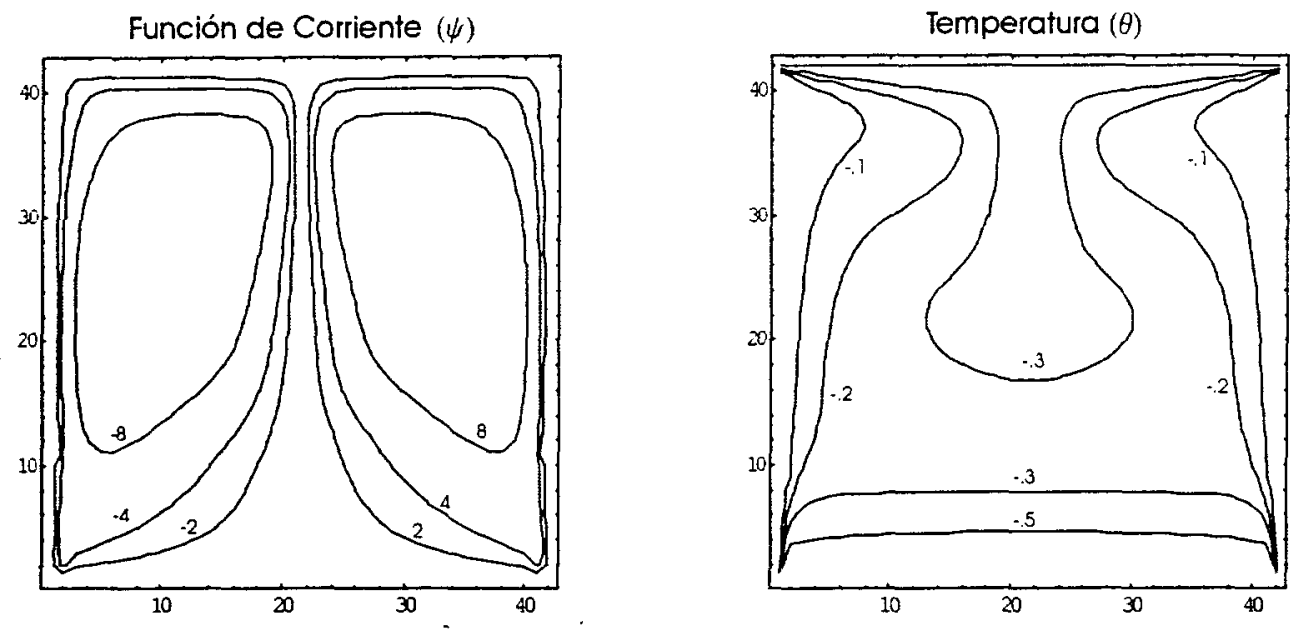

Figura 5

Contornos de la función de corriente y de la temperatura para $t=0.038$

Finalmente, en la figura 5, las celdas laterales han seguido creciendo hasta lograr vencer a los otros vórtices, con lo cual sólo se tienen dos celdas, una a cada lado de la línea central, circulando en sentidos opuestos. En la gráfica de la temperatura, ésta se ha estabilizado y el fluido es ahora frío en el centro de la cavidad y disminuyendo conforme nos acercamos hacia las fronteras superior o inferior, mientras que la temperatura aumenta si estamos cerca de las paredes, las cuales se mantienen cálidas. 


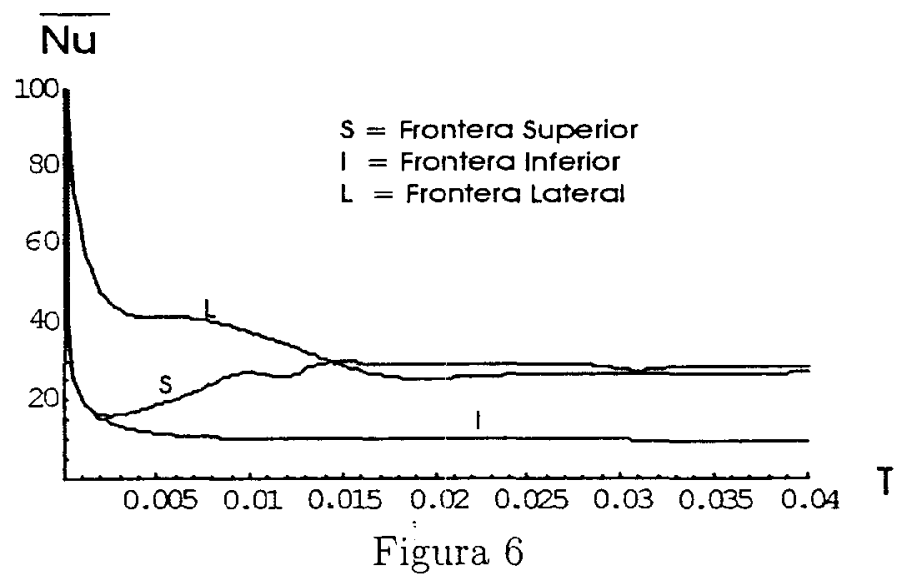

Número de Nusselt promedio

para $R=1.000$ y $\theta=-0.5$

Por otra parte, la figura 6 presenta la gráfica del número de Nusselt promedio, a lo largo de cada una de las fronteras que limita la cavidad, para estas condiciones.

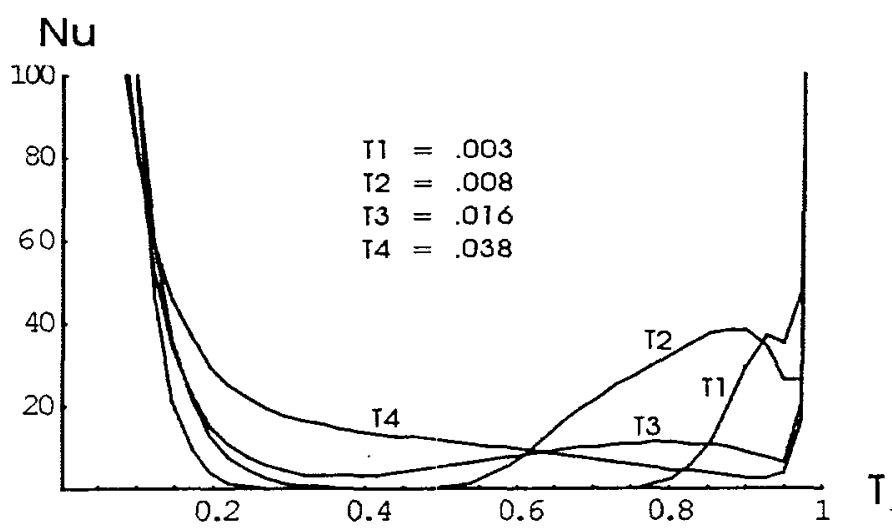

Figura 7

Número de Nusselt a lo largo de la superficie lateral para $R=1,000$ y $\theta=-0.5$

Como se observa de la gráfica, tanto en la frontera inferior como en la superior, el número de Nusselt promedio inicialmente es infinito, lo cual se debe a la discontinuidad de la temperatura en éstas superficies, mientras que en las paredes el número de Nusselt promedio también es infinito al principio debido a que la temperatura promedio del fluido es la misma que 
la de la pared. A lo largo de cada superficie, observamos que el número de Nusselt promedio decrece conforme se desarrolla el flujo, sin embargo, el número de Nusselt en la frontera superior presenta un aumento cuando los vórtices mayores vencen a los pequeños, y este incremento es el resultado de las mayores velocidades que se alcanzan asociadas con los vórtices más grandes.

En la figura 7 mostramos los números locales de Nusselt a lo largo de una de las paredes $(x=0)$ para cuatro diferentes tiempos en donde observamos que los valores son muy grandes cerca de la tapa inferior, porque el flujo que se encuentra sobre esta superificie choca contra la pared y se vuelve hacia arriba, mientras que la parte superior de la pared muestra el efecto provocado por lo vórtices que inicialmente se desarrollan en la esquina, $t=.003$ y $t=.008$, de tal forma que el fluido tiende a fluir hacia el centro de la pared; en tiempos posteriores, $\mathrm{t}=.016 \mathrm{y} t=.038$, éste tiende a disminuir debido a que el vórtice de la esquina inferior crece hasta llenar la mitad de la cavidad, con lo que el número de Nusselt decrece uniformemente cuando el fluido se eleva desde el fondo de la cavidad.

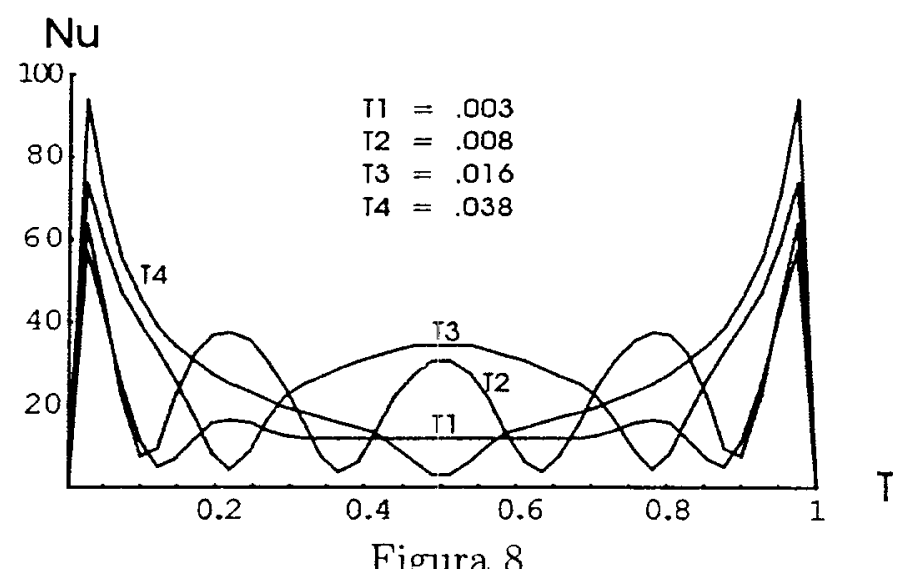

Número de Nusselt a lo largo de la superficie superior para $R=1,000$ y $\theta=-0.5$

A lo largo de la superficie superior, fig. 8, el número local de Nusselt es grande cerca de la pared, pues como se mencionó anteriormente, esto es debido a que la corriente asciende por la pared y choca con la superficie superior. Cuando $t=.008$ se crean vórtices secundarios hacia la cuarta parte de la tapa que se van alejando de ésta, lo cual repercute en altos y bajos números de Nusselt respectivamente. 


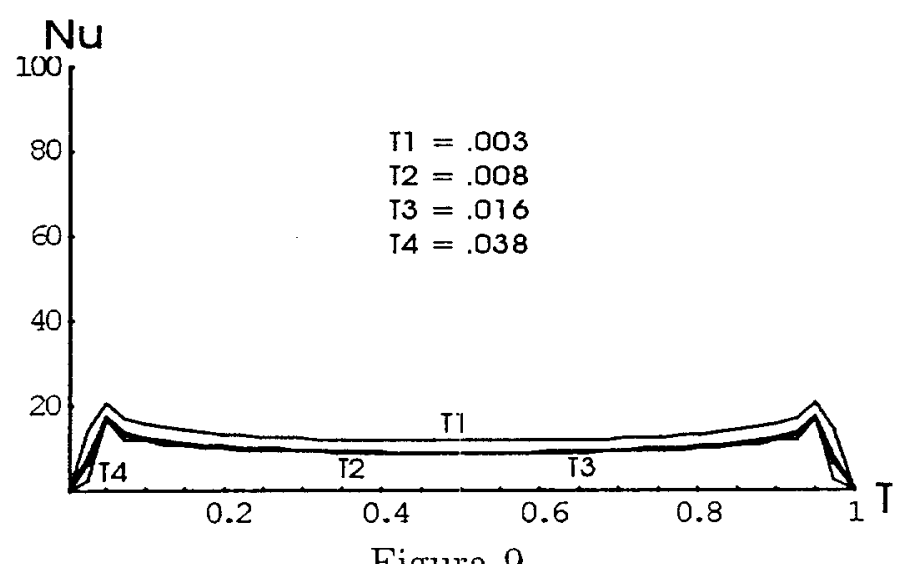

Figura 9

Número de Nusselt a lo largo de la superficie inferior para $R=1,000$ y $\theta=-0.5$

En la frontera inferior, fig. 9, los valores del número de Nusselt son notoriamente más pequeños y estables ya que el flujo es relativamente lento en el fondo de la cavidad y paralelo a ésta, y los valores más altos se alcanzan cerca de la pared, lo cual resulta de que la velocidad del flujo en esa zona toma sus valores más altos.

Caso II) $\theta_{A}=+0.5, \quad R=10,000, \quad h=\frac{1}{80}, \quad \Delta t=.00001$

El sistema recibe calor a través de la superficie superior y de las paredes, estas últimas a menor temperatura que la primera, y se enfría en la frontera inferior. El fluido se desarrolla hacia un estado estacionario para $t>0.03$, donde se observa de la gráfica de la función corriente, fig. 10, que el fluido, que es enfriado en la frontera inferior de la cavidad y calentado en las paredes, circula ascendiendo en capas flotantes a lo largo de éstas hasta la mitad del plano y se enfría nuevamente al llegar al fondo.

Mientras tanto, en la parte superior el fluido es calentado en la tapa y puesto que las paredes se encuentran a menor temperatura que aquella, esto provoca que el fluido se enfríe a lo largo de las fronteras laterales, con lo que la fuerza de flotación lo empuja hacia àbajo, produciendo también un vórtice en cada esquina superior.

Así se obtienen 4 vórtices, uno en cada esquina, en donde los vórtices de las esquinas superior izquierda e inferior derecha giran en sentido positivo mientras que los vórtices de las esquinas superior derecha e inferior izquierda lo hacen en sentido opuesto, es decir en sentido negativo; es por esta razón 

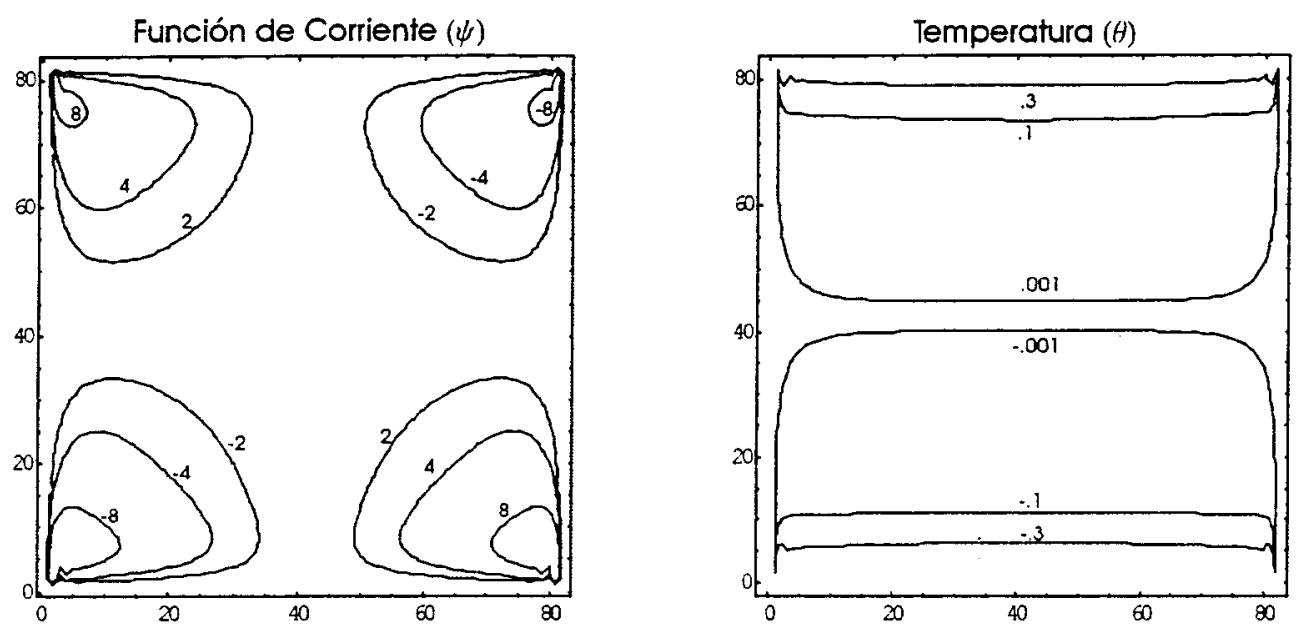

Figura 10

Contornos de la función de corriente y de la temperatura para $t=0.03$

que la pareja de vórtices derechos no se funde finalmente en una sola celda, pues giran en sentidos opuestos, y de igual forma sucede con la pareja de vórtices izquierdos.

La distribución de temperatura muestra que el fluido mantiene una temperatura más cálida en la mitad superior y más fría en la mitad inferior, y estas diferencias se atenúan conforme nos acercamos hacia el centro de la cavidad.

Caso III) $\theta_{A}=0.0, \quad R=10,000, \quad h=\frac{1}{80}, \quad \Delta t=.00001$

La figura 11 muestra que el fluido se enfría en la frontera inferior y se calienta tanto en las paredes como en la frontera superior (las cuales se encuentran a la misma temperatura que el fluido inicial), entonces el equilibrio del fluido en la mitad superior de la cavidad no se ve alterado, por tal razón no existe flujo en dicha parte. En la mitad inferior se presenta un vórtice en cada esquina, de manera semejante a la respectiva gráfica del caso II, es decir el fluido frío del fondo se va calentado a lo largo de las paredes, hasta la mitad de la cavidad, y se vuelve a enfriar al llegar nuevamente al fondo, de tal manera que los vórtices de cada esquina circulan en sentidos opuestos. Es importante hacer notar la similitud de los vórtices inferiores en los casos II y III, lo cual indica que la presencia de las celdas superiores en el caso anterior prácticamente no ejercen efecto sobre las celdas inferiores. 

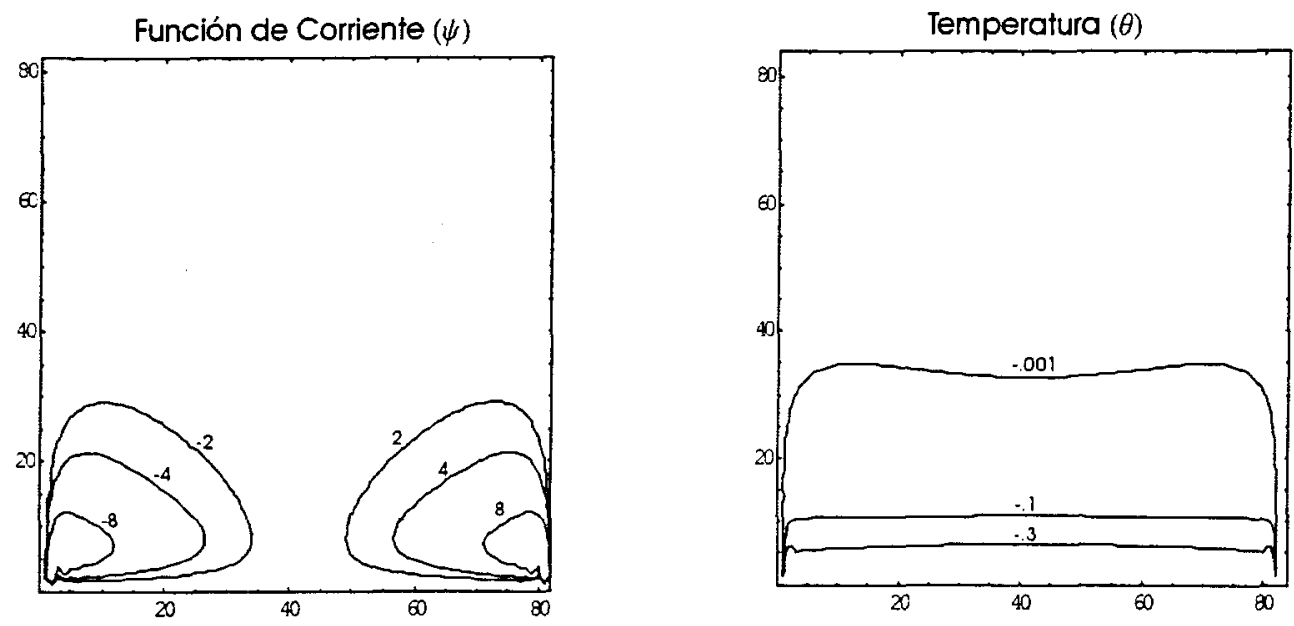

Figura 11

Contornos de la función de corriente y de la temperatura para $t=0.011$

Por esta misma razón, la gráfica de la temperatura prácticamente no muestra diferencia con respecto a la mitad inferior de la correspondiente gráfica del caso II, mientras que en la mitad superior se mantiene prácticamente a la misma temperatura inicial.

Caso IV) $\theta_{A}=-0.5, \quad R=10,000, \quad h=\frac{1}{80}, . \quad \Delta t=.000005$

En este caso al contrario de los anteriores no se alcanza un estado estacionario, en su lugar el flujo se desarrolla hacia una solución de tipo casi periódico.

Las siguientes figuras muestran las gráficas de la función corriente y de la temperatura para cuatro diferentes tiempos, de inicio y fin de un casi-período.

Al igual que en el caso I, el calentamiento se produce en las paredes, mientras que el fluido se enfría a través de las fronteras superior e inferior.

En la gráfica de la función corriente, la característica principal, es que obtenemos dos largas celdas que ocupan casi la totalidad de la cavidad y que rotan en sentido opuestos. Cerca de la tapa, las celdas presentan disturbios debido a que el fluido se enfría en esa superficie, fig. 12.a, y comienza a sumergirse, sin embargo las siguientes gráficas muestran cómo estas distorsiones van disminuyendo a medida que el flujo circula hacia la línea central de la cavidad, fig. 12.c , para posteriormente aumentar las distorsiones nuevamente, figs. 12.e y 12 .g; mientras esto sucede en la mitad superior, en 

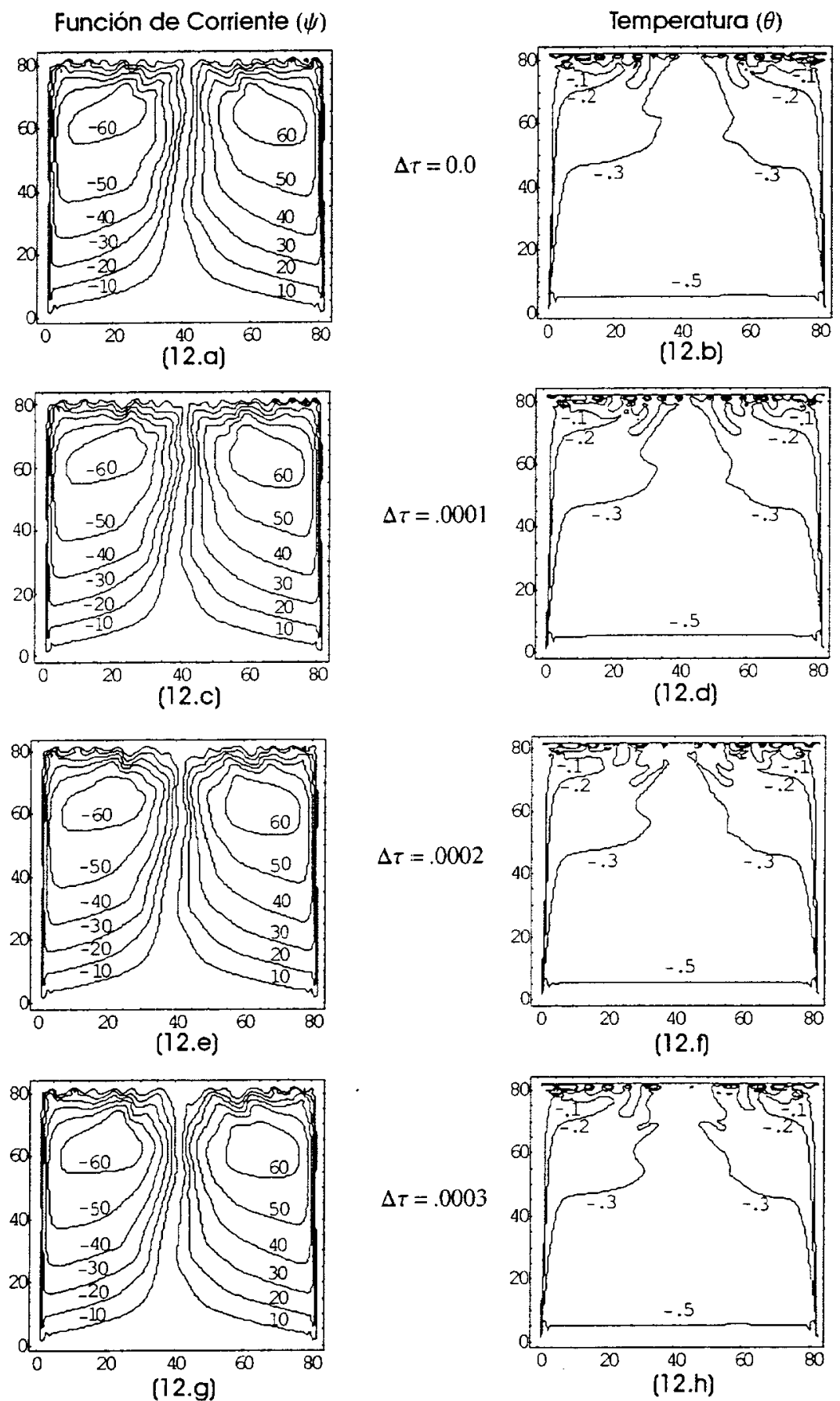

Figura 12

Contornos de la función de corriente y de la temperatura durante un ciclo, cuyo período $\Delta t=0.0004$ 
la mitad inferior la gráfica permanece sin cambios notorios, con lo que las velocidades en esta parte de la cavidad permaneacen prácticamente constantes.

Las correspondientes gráficas para la temperatura, muestra que el fluido se ha enfriado, lo cual es más notorio en las fronteras superior e inferior así como en el centro y aumenta conforme nos acercamos a las superficies laterales.

Es importante notar el hecho de que las gráficas de la función corriente no es totalmente antisimétrica, por las claras discrepancias que se observan en la parte superior de la mismas, si bien es cierto el hecho de que las celdas obtenidas rotan en sentidos opuestos. De la misma manera, las gráficas de la temperatura tampoco son simétricas en la parte superior, y estas diferencias son igualmente notorias. El período estimado es de .0004 .

Este es el único caso en el que los resultados que obtuvimos no coinciden del todo con los reportados por Christopher, ya que él indica que obtuvo estados periódicos, determinando el periodo en 0.00048 , y aunque no menciona la simetría de la temperatura ni la antisimetría de la función corriente específicamente para este caso, supone del análisis primario que hace del problema general que las satisfacen, lo cual para nosotros no fue así, ya que como se observa de las gráficas que obtuvimos se presentan claras diferencias entre la primera y la segunda mitad de la cavidad.

\subsection{Condiciones de Frontera Mixtas}

El mismo problema fue resuelto considerando diferentes tipos de condiciones de frontera para la temperatura al mismo tiempo que se mantiene siempre condiciones de frontera Dirichlet para la función corriente. Los resultados obtenidos consideramos que fueron satisfactorios; a continuación presentamos algunos de estos resultados cuando se tienen condiciones de frontera mixtas para la temperatura y Dirichlet para la función corriente.

Caso I) $\quad R=1,000, \quad h=\frac{1}{30}, \quad \Delta t=.0002$

Las condiciones de frontera que se satisfacen en este caso son:

$$
\begin{aligned}
& \theta=0 \text { en } x=0 \quad y \quad x=1 \\
& \frac{\partial \theta}{\partial n}=1 \text { en } z=0 \\
& \frac{\partial \theta}{\partial n}=0 \text { en } z=1 \\
& \psi=0 \text { en } x=0 \quad y \quad x=1 \\
& \psi=0 \text { en } z=0 \text { y } y=1
\end{aligned}
$$


A partir de las condiciones de frontera,se tiene que existe transferencia de calor desde el exterior hacia el sistema a través del fondo de la cavidad, lo cual significa que se introduce calor al sistema, mientras que en las superficies laterales se tiene una temperatura constante y la frontera superior es adiabática por lo cual el sistema se mantiene aislado en esta superficie.
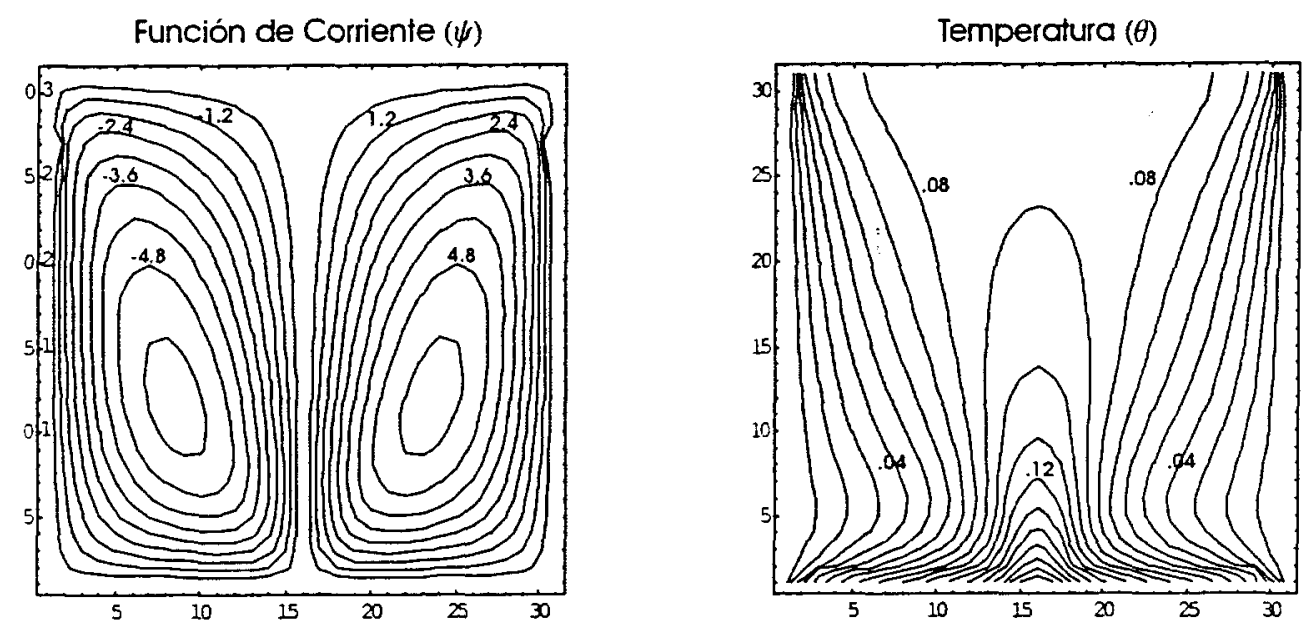

Figura 13

Contornos de la función de corriente y de la temperatura para $t=0.105$

En la fig. 13 se muestran algunos contornos para las gráficas de la temperatura y para la función corriente cuando se ha llegado al estado estacionario $(\mathrm{t}=.105)$. Observamos que se sigue manteniendo, en este caso, la antisimetría de la función corriente y la simetría para la temperatura. La gráfica de la temperatura muestra que el mayor calentamiento se da en el centro del fondo de la cavidad, razón por la cual el fluido caliente del centro se eleva y va enfriándose en la parte superior de la cavidad y posteriormente cae por las paredes hasta el fondo donde vuelve a calentarse, por ello en la gráfica de la función corriente se observan dos celdas que ocupan el total de la cavidad y que rotan en sentidos opuestos.

Caso II) $\quad R=100, \quad h=\frac{1}{20}, \quad \Delta t=.0002$

Las condiciones de frontera que se satisfacen en este caso son: 


$$
\begin{aligned}
& \theta=1 \text { en } x=0 \\
& \theta=0 \text { en } x=1 \\
& \frac{\partial \theta}{\partial n}=0 \text { en } z=0 \text { y } \begin{array}{l} 
\\
\psi=0
\end{array}=1 \\
& \psi=0 \text { en } x=0 \text { y } x=1 \\
& \psi=0 \text { en } z=0 \text { y } z=1
\end{aligned}
$$

En este caso, las fronteras superior e inferior son adiabáticas, y las superficies laterales mantienen temperaturas constantes distintas. Debido a las condiciones de frontera para la temperatura, no esperamos que se conserven las propiedades de la antisimetría y simetría para la función corriente y para la temperatura, respectivamente.
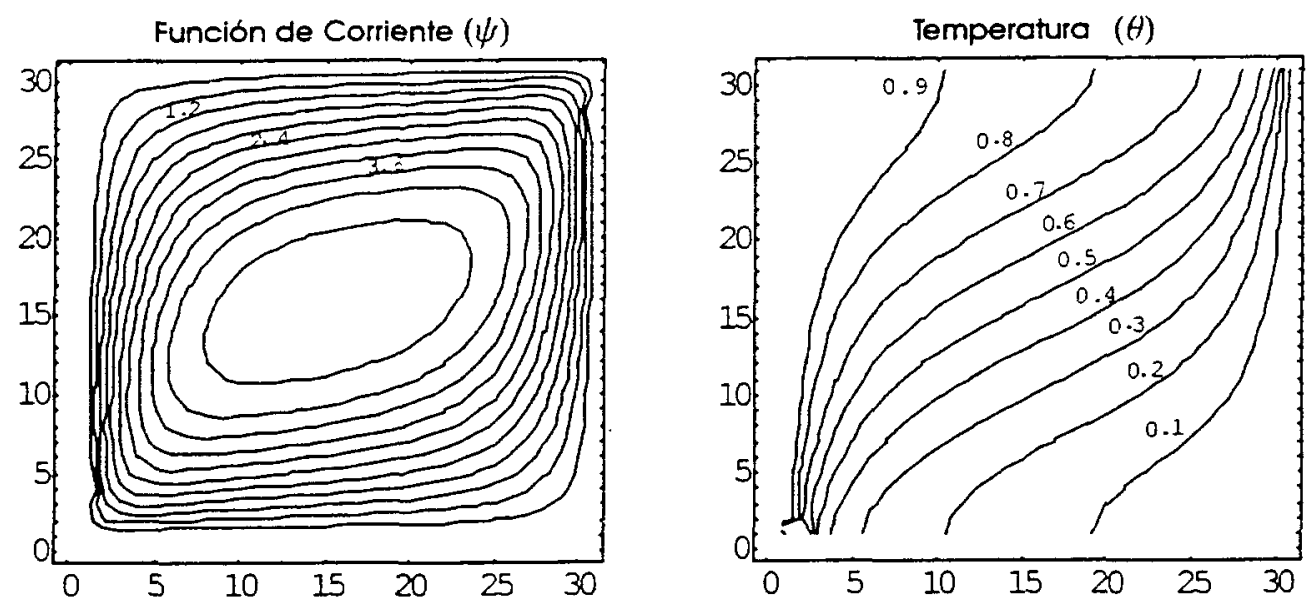

Figura 14

Contornos de la función de corriente y de la temperatura para $t=0.094$

En el estado estacionario, $t=.094$, se obtiene en la gráfica de la función corriente una sola celda circulante en toda la cavidad; además las gráficas corroboran que no existe antisimetría en la función corriente ni simetría en la gráfica de la temperatura.

Los resultados obtenidos en el caso de condiciones de frontera mixtas son concordantes con aquellas obtenidas por el Dr. en Ciencias Hugo Jiménez Islas en su trabajo de tesis doctoral.

Finalmente, mencionamos que los cálculos numéricos de este trabajo se realizaron en la computadora (computadora IRIX de SGI), la cual cuenta 
con 2 procesadores R 4000, $256 \mathrm{MB}$ en RAM y $17.5 \mathrm{~GB}$ en disco duro, del Laboratorio de Supercómputo de la UAM-I. 


\section{Capítulo 6}

\section{Conclusiones}

Resumen: Se evalúan los resultados obtenidos al considerar condiciones Dirichlet y se hace una revisión comparativa con los resultados obtenidos por Christopher. Posteriormente, se presenta una síntesis de las principales características obtenidas en los resultados correspondientes a las condiciones mixtas. Finalmente, se hace una breve reseña sobre otros problemas, con características similares a los presentados aquí, que pueden ser resueltos con el mismo método propuesto en este trabajo.

\subsection{Condiciones de Frontera Dirichlet}

Los resultados que obtuvimos nos indican que:

a) Cuando el número de Darcy-Raleigh es pequeño $(R=1000)$ y las condiciones de frontera son inestables, esto es, cuando la superficie superior se encuentra a menor temperatura que las superficies laterales, el flujo se desarrolla hasta llegar a un estado estacionario en el cual se tiene una sola celda a cada lado de la línea central, las cuales ocupan el total de la cavidad y rotan en sentidos opuestos.

b) Cuando el número de Darcy-Raleigh es grande $(R=10,000)$ y para los casos en que la frontera superior se encuentra a mayor o igual temperatura que las fronteras laterales (condiciones de frontera estables) el desarrollo de flujo también es hacia un estado estacionario, en el cual se obtienen dos celdas rotando en sentidos opuestos, una a cada lado de la línea central cuando la frontera superior tiene la misma temperatura que las fron- 
teras laterales y cuatro celdas rotando en contrasentido una con respecto a la otra, dos a cada lado de la línea central vertical, cuando la superficie superior mantiene una temperatura mayor que la de las superficies laterales.

c) Cuando $R$ es grande $(R=10000)$ y las condiciones de frontera son inestables, donde la superficie superior se encuentra más fría que las superficies laterales, el flujo adquiere un carácter de tipo casi periódico en donde las dos celdas obtenidas, cada una ocupando una mitad de la cavidad y rotando en contraposición una con respecto a la otra, tienen un período aproximado de crecimiento y decaimiento del flujo que circula a lo largo de la superficie superior cuando éste se enfría y posteriormente cae hacia el centro de la cavidad, donde se encuentra el fluido más tibio.

Estos resultados concuerdan, en general, con los reportados por Christopher, sin embargo, las principales discrepancias son:

a) en los casos en que se llega a obtener un estado estacionario, los tiempos obtenidos por nosotros para alcanzar tanto los mismos estados transitorios indicados por Christopher como para los estados estacionarios son menores en todos los casos,

b) mientras que Christopher reporta una solución de tipo periódico cuando $\mathrm{R}=10,000$ y condiciones de frontera inestables, nosotros obtenemos una solución casi periódica, pues existen ligeras diferencias entre lo que pudieramos llamar período de la solución; además, en el desarrollo del flujo se observan algunas discrepancias entre lo que se obtiene en una mitad de la cavidad y la otra que provocan que se pierda un poco de la antisimetría de la función corriente, y por la misma razón, tampoco se satisface del todo la simetría de la gráfica para la temperatura.

\subsection{Condiciones de Frontera Mixtas}

En los dos casos presentados en este trabajo, los resultados del estado estacionario concuerdan bastante bien con los reportados por el Dr. Jiménez Islas, el cual resolvió los mismos problemas pero considerando las ecuaciones estacionarias respectivas, es decir no dependientes del tiempo, usando un método de colocación ortogonal.

a) Cuando consideramos el valor del número de Darcy-Raleigh de 1,000 
y las condiciones de frontera indican que las paredes tienen temperatura constante $(\theta=0)$, la superficie superior está aislada y en la superficie inferior existe ingreso de calor $\left(\frac{\partial \theta}{\partial n}=1\right)$ se llega a un estado estacionario en donde se obtiene una celda a cada lado de la mitad de la cavidad, las cuales se limitan mutuamente pues rotan en sentidos opuestos, mientras que temperatura del fluido es mayor en el fondo y centro de la cavidad.

b) Cuando el valor de $R$ es 100 y tanto la superficie superior como la inferior están aisladas mientras que las fronteras laterales se mantienen a temperatura constante, pero distinta una de la otra, en el estado estacionario se obtiene una sola celda llenando la cavidad.

Los resultados obtenidos son muy satisfactorios y prácticamente no existen discrepancias con aquellos presentados por el Dr. Jiménez. Sin embargo, es importante aclarar que se hicieron algunos ajustes en las ecuaciones, adimensionales, para que fueran equivalentes a aquellas que resolvió el Dr. Jiménez, lo cual también se verá reflejado en los resultados,

Estos ajustes están relacionados básicamente con signos en las ecuaciones respectivas a la función corriente:

a) En la definición de la función corriente se consideraron las siguientes relaciones:

$$
\begin{aligned}
& u=-\frac{\partial v}{\partial z} \\
& \nu=\frac{\partial v}{\partial x} .
\end{aligned}
$$

con lo cual las ecuaciones de momento y de energía toman la forma

$$
\left.\begin{array}{rl}
\frac{1}{R} \nabla^{2} \psi & =\frac{\partial \theta}{\partial x} \\
\frac{\partial \theta}{\partial t}-\nabla^{2} \theta+\mathrm{v} \cdot \nabla \theta & =0
\end{array}\right\}
$$

donde

$$
\mathbf{v}=(u, v)=\left(-\frac{\partial \psi}{\partial z}, \frac{\partial \psi}{\partial x}\right)
$$

b) Al resolver las ecuaciones obtenidas con esta definición de la función corriente y condiciones de frontera mixtas para la temperatura, cuyos resultados son los presentados anteriormente, observamos que en el caso en 
que la frontera superior sea adiabática y en la frontera inferior se satisfaga la condición $\frac{\partial \theta}{\partial n}=1$, las gráficas de la función corriente y de la temperaratura son las mismas que cuando se resuelven con las ecuaciones originales. Sin embargo, en el caso de la función corriente, donde se obtienen dos celdas en la cavidad, los valores obtenidos son los mismos pero con signos opuestos, en el caso de la gráfica de la temperatura no hubo ninguna diferencia.

c) En el caso en que las fronteras laterales son adiabáticas y con las ecuaciones originales, tanto la gráfica de la temperatura como de la función corriente presentaron los mismos valores positivos, pero la dirección de las líneas fue opuesta en ambos casos, es decir en el caso de la temperatura parecieran iniciar en la esquina superior izquierda y dirigirse hacia la esquina inferior derecha, análogamente sucede con los contornos de la función corriente.

\subsection{Sugerencias para la Resolución de Pro- blemas Similares}

En la sección anterior se observaron ya un par de casos, que son similares al original y que pueden ser resueltos con el método propuesto en este trabajo, en general algunos otros problemas, relacionados con flujos en medios porosos, que pueden ser resueltos por este método tienen las siguientes características:

a) Problemas donde al ser adimensionadas las ecuaciones respectivas, tienen la misma forma que las presentadas en el presente trabajo, pero con condiciones de frontera Dirichlet, Neumann o mixtas. En este caso, realizamos diversas pruebas con diferentes condiciones de frontera y los resultados fueron satisfactorios.

b) Problemas donde las ecuaciones adimensionales de energía y de momento sean

$$
\left.\begin{array}{rl}
-\frac{1}{R} \nabla^{2} \psi & =\frac{\partial \theta}{\partial x} \\
\frac{\partial \theta}{\partial t}-\frac{1}{P_{r}} \nabla^{2} \theta+\mathrm{v} \cdot \nabla \theta & =0
\end{array}\right\}
$$

donde Pr denota el número de Prandalt, el cual es un parámetro impor- 
tante en el análisis de transferencjar de calor y $\mathrm{v}=(u, v)=\left(\frac{\partial \psi}{\partial z},-\frac{\partial \psi}{\partial x}\right)$. Algunas pruebas fueron realizadas para diferentes valores del número de Prandalt y con condiciones de front cra de tipo dirichlet, y obtuvimos buenos resultados; consideramios que es posible resolver de manera satisfactoria estas mismas ecuaciones con otro tipo de condiciones de frontera. 


\section{Apéndice A}

\section{Variables dimensionales y adimensionales}

Las cantidades dimensionales y adimensionales que utilizamos tanto en el problema continuo como en el discreto son las siguientes:

A -ancho de la cavidad $(m)$.

$B$-altura de la cavidad $(m)$.

$c_{p}$-calor específico $\left({ }^{o} \mathrm{~K} \mathrm{~m}^{2} / \mathrm{s}^{2}\right)$.

$\mathrm{g}$-aceleración gravitacional $\left(\mathrm{m} / \mathrm{s}^{2}\right)$.

$\mathrm{i}$-índice para la dirección $\mathrm{x}$.

$\mathrm{j}$-índice para la dirección $\mathrm{z}$.

L -razón ancho-largo de la cavidad .

$k$-permeabilidad del medio poroso $\left(m^{2}\right)$.

$\mathrm{P}$-presión $(\mathrm{Pa})$.

$\mathrm{R}$-número de Darcy-Raleigh .

$\mathrm{T}$-temperatura $\left({ }^{\circ} \mathrm{K}\right)$.

$\mathrm{T}_{e}$-temperatura de referencia $\left({ }^{\circ} \mathrm{K}\right)$.

$\mathrm{T}_{u}$-temperatura de las paredes laterales de la cavidad $\left({ }^{\circ} K\right)$.

$\mathrm{T}_{1}$-temperatura de la pared superior de la cavidad $\left({ }^{\circ} \mathrm{K}\right)$.

$\mathrm{T}_{2}$-temperatura de la pared inferior de la cavidad $\left({ }^{\circ} \mathrm{K}\right)$.

$\mathrm{t}$-tiempo adimensional .

$t^{\prime}$-tiempo $(s)$.

$\Delta t$-paso de tiempo adimensional. 
$\mathrm{u}$-velocidad adimensional en la dirección $\mathrm{x}$.

$\mathrm{u}^{\prime}$-velocidad en la dirección $\mathrm{x}(\mathrm{m} / \mathrm{s})$.

$\mathrm{u}_{\max }$-velocidad máxima en la dirección $\mathrm{x}(\mathrm{m} / \mathrm{s})$.

$\mathrm{v}$-velocidad adimensional en la dirección $\mathrm{z}$.

$v^{\prime}$-velocidad en la dirección $z(\mathrm{~m} / \mathrm{s})$.

$\mathrm{x}$-coordenada horizontal adimensional.

$x^{\prime}$-coordenada horizontal $(m)$.

$\mathrm{h}$-ancho de la malla.

$z$-coordenada vertical adimensional.

$z^{\prime}$-coordenada vertical $(m)$.

$\beta$-coeficiente de expansión térmica $\left(1 /{ }^{\circ} K\right)$.

$\theta$-temperatura adimensional.

$\theta_{A}$-temperatura adimensional en la frontera superior.

$\bar{\theta}$-temperatura promedio adimensional.

$\kappa$-coeficiente de difusividad térmica $\left(\mathrm{m}^{2} / \mathrm{s}\right)$.

$\mu$-coeficiente de viscosidad dinámica $(\mathrm{kg} / \mathrm{m} \mathrm{s})$.

$\rho$-densidad $\left(\mathrm{kg} / \mathrm{m}^{3}\right)$.

$\rho_{e}$-densidad de referencia $\left(\mathrm{kg} / \mathrm{m}^{3}\right)$.

$\eta$-conductividad térmica del fluido $\left(m \mathrm{~kg} / \mathrm{s}^{3}{ }^{\circ} \mathrm{K}\right)$.

$\psi$-función corriente adimensional.

$\psi^{\prime}$-función corriente $\left(\mathrm{m}^{2} / \mathrm{s}\right)$. 


\section{Apéndice B}

\section{Método Iterativo SOR por Bloques}

Cuando aplicamos métodos de diferencias finitas para encontrar una solución aproximada de una ecuación diferencial parcial, generalmente se requiere resolver un sistema de ecuaciones lineales, $\mathbf{A x}=\mathbf{b}$, en el cual la matriz $\mathbf{A}$ asociada al sistema es rala y presenta una estructura de bloques, esto es

$$
\mathbf{A}=\left(\begin{array}{ccc}
\mathbf{A}_{11} & \cdots & \mathbf{A}_{1 N} \\
\vdots & \vdots & \vdots \\
\mathbf{A}_{N 1} & \cdots & \mathbf{A}_{N N}
\end{array}\right)
$$

donde cada $\mathbf{A}_{j j}$ es matriz cuadrada, con $\mathbf{j}=1, . ., \mathrm{N}$.

Para resolver este sistema lineal, generalmente elegimos un método iterativo, ya que los métodos usuales de eliminación tienden a llevarnos durante el proceso a matrices intermedias más o menos densas, lo cual incrementaría considerablemente el número de operaciones necesarias para encontrar la solución.

Por esta razón, y además debido a la estructura de la matriz $\mathbf{A}$, conviene utilizar un método iterativo por bloques; para nuestro caso optamos por el método de sobre-relajación por bloques el cual expresamos como

$$
\begin{aligned}
& \mathrm{A}_{j j} \mathbf{x}^{i+1}=\omega \mathrm{b}_{j}-\omega \sum_{k<j} \mathbf{A}_{j k} \mathbf{x}_{k}^{i+1}-\omega \sum_{k>j} \mathbf{A}_{j k} \mathbf{x}_{k}^{i}+(1-\omega) \mathbf{A}_{j j} \mathbf{x}^{i} \\
& \text { cons: } \quad j=1, \ldots, N ; \quad i=1,2, \ldots
\end{aligned}
$$


donde los vectores $\mathbf{x}$ y $\mathbf{b}$ se particionan de manera similar a $\mathbf{A}$ y $\omega$ es el parámetro de relajación.

Como se observa, en cada paso debemos resolver $\mathrm{N}$ sistemas de ecuaciones lineales de la forma $\mathbf{A}_{j j} z=y, \quad j=1, \ldots, N$. Este proceso lo realizamos haciendo la descomposición de Cholesky para cada matriz $\mathbf{A}_{j j}$ y sustituyendola en el sistema, así si

$$
\mathbf{A}_{j j}=\mathbf{L}_{j} \mathbf{L}_{j}^{t}
$$

entonces

$$
\mathrm{L}_{j} \mathbf{u}=\mathbf{y}, \quad \mathrm{L}_{j}^{t} \mathbf{z}=\mathbf{u}
$$

en donde realizando directamente primero la sustitución hacia adelante en el primer sistema y sustituyendo, después, el valor encontrado para $u$ en el segundo sistema y haciendo la sustitución hacia atrás en el segundo sistema habremos determinado el valor de $\mathbf{z}$.

Recordemos que en nuestro problema la matriz A tiene la siguiente estructura de bloques

$$
\mathbf{A}=\frac{1}{h^{2}}\left(\begin{array}{ccccc}
\mathrm{A}_{1} & -\mathrm{I}_{1} & \mathrm{O}_{1} & \cdots & \mathrm{O}_{1} \\
-\mathrm{I}_{1} & \ddots & \ddots & \ddots & \vdots \\
\mathrm{O}_{1} & \ddots & \ddots & \ddots & \mathrm{O}_{1} \\
\vdots & \ddots & \ddots & \ddots & -\mathrm{I}_{1} \\
\mathrm{O}_{1} & \cdots & \mathrm{O}_{1} & -\mathrm{I}_{1} & \mathrm{~A}_{1}
\end{array}\right)
$$

donde como se observa, las matrices $\mathbf{A}_{j j}$ indicadas anteriormente son todas iguales para $\mathrm{j}=1, \ldots, \mathrm{N}, \mathrm{y}$ la denotamos por $\mathbf{A}_{1}$, cuya estructura es

$$
\mathbf{A}_{1}=\left(\begin{array}{ccccc}
4 & -1 & 0 & \cdots & 0 \\
-1 & \ddots & \ddots & \ddots & \vdots \\
0 & \ddots & \ddots & \ddots & 0 \\
\vdots & \ddots & \ddots & \ddots & -1 \\
0 & \cdots & 0 & -1 & 4
\end{array}\right)
$$

mientras que :

$\mathrm{I}_{1}=$ matriz identidad de orden $N \times N$,

$\mathrm{O}_{1}=$ matriz nula de orden $N \times N$. 
Claramente tanto $\mathbf{A}$ como $\mathbf{A}_{1}$ son simétricas, y ya mencionamos que son definidas positivas. Además, la estructura tridiagonal en (B.5) obviamente simplifica significativamente a (B.2). 


\section{Apéndice $\mathrm{C}$}

\section{Fishpack}

Fishpak es un paquete de prograrración en Fortran [18], mediante el cual es posible resolver ecuaciones diferenciales parciales elípticas separables de la forma:

$$
A F(X) * U_{X X}+B F(X) * U_{X}+C F(X) * U+U_{Y Y}=G(X, Y)
$$

usando una aproximación de diferencias finitas, de segundo o de cuarto orden, sobre un rectángulo $(A \leq X \leq B, C \leq Y \leq D)$ y para cualesquiera combinación de condiciones de frontera periódicas o mixtas.

Las posibles condiciones de frontera son:

En la dirección $\mathrm{X}$ :

(0) Periódica, $U(X+B-A, Y)=U(X, Y)$ para toda $X, Y$.

(1) Dirichlet, $\mathrm{U}(\mathrm{A}, \mathrm{Y}), \mathrm{U}(\mathrm{B}, \mathrm{Y})$, especificadas para toda $\mathrm{Y}$.

(2) Mixtas, $\mathrm{U}(\mathrm{A}, \mathrm{Y}), \frac{D U(B, Y)}{D X}+B E T A * U(B, Y)$ son especificadas para toda $\mathrm{Y}$.

(3) Neumann, $\frac{D U(A, Y)}{D X}+A L P H A * U(A, Y), \frac{D U(B, Y)}{D X}+B E T A * U(B, Y)$ especificadas para toda $\mathrm{Y}$.

(4) Mixtas, $\frac{D U\left(A, Y^{\prime}\right)}{D X}+A L P H A * U(A, Y), \mathrm{U}(\mathrm{B}, \mathrm{Y})$ especificadas para toda Y. 
En la dirección Y:

(0) Periódica, $U(X, Y+D-C)=U(X, Y)$ para toda $\mathrm{X}, \mathrm{Y}$.

(1) Dirichlet, U(X,C), U(X.D). especificadas para toda X.

(2) Mixtas, U(X,C), $\frac{D U(X, D)}{D Y}$ especificadas para toda X.

(3) Neumann, $\frac{D U(X, C)}{D Y}, \frac{D U(X, D)}{D Y}$ especificadas para toda $X$.

(4) Mixtas, $\frac{D U(X . C)}{D Y}, \mathrm{U}(\mathrm{X}, \mathrm{D})$ especificadas para toda $\mathrm{X}$.

El formato para llamar a este paquete es:

CALL DEPX4 (IORDER,A,B,M, MBDCND,BDA,ALPHA, BDB,BETA, C,D, N,NBDCND,BDC,BDD,COFX,GRHS, USOL,IDMN,W, PERTRB, IERROR)

en donde los respectivos argumentos indican :

IORDER

$=2$ si se desea una aproximación de segundo orden.

$=4$ si se desea una aproximación de cuarto orden.

$A, B=$ rango de la variable $X$.

$M=$ número de subdivisiones del intervalo $[A, B] . M$ debe ser menor que IDMN y mayor que 5 .

MBDCND = toma el valor de: $0.1 .2,3$ ó 4 dependiendo de las condiciones de frontera para $X$.

$\mathrm{BDA}=$ arreglo unidimensional de longitud $N+1$ que especifica los valores de $\frac{D U(A, Y j)}{D X}+B E T A * U(A, Y j)$, considerando $j=1, \ldots, N+1$; cuando $\mathrm{MBDCND}=3$ ó 4 . Si MBDCND toma cualquier otro valor, BDA es un parámetro mudo.

ALPHA = escalar que multiplica a la solución en caso de tener una condición de frontera mixta en $\mathrm{X}=\mathrm{A}$. Si MBDCND $=3$ ó 4 entonces ALPHA es un parámetro mudo.

$\mathrm{BDB}=$ arreglo unidimensional de longitud $N+1$ que especifica los valores de $\frac{D U(B, Y j)}{D X}+B E T A * U(B, Y j)$, considerando $j=1, \ldots, N+1$; cuando do $M B D C N D=2$ ó 3. Si MBDCND toma cualquier otro valor, BDB es un pará metro mudo. 
BETA = escalar que multiplica a la solución en caso de tener una condición de frontera mixta en $\mathrm{X}=\mathrm{B}$. Si $\mathrm{MBDCND}=2$ ó 3 entonces BETA es un parámetro mudo.

$\mathrm{C}, \mathrm{D}=$ rango de la variable $\mathrm{Y}$.

$N=$ número de subdivisiones del intervalo $[C, D]$. $N$ debe ser mayor que

4.

NBDCND = toma el valor de: $0,1,2,3$, ó 4 dependiendo de las condiciones de frontera para $Y$.

$\mathrm{BDC}=$ arreglo unidimensional de longitud $\mathrm{M}+1$ que especifica los valores de $\frac{D U(X i . C)}{D Y}$, considerando $i=1, \ldots, M+1$, cuando NBDCND $=3$ ó 4 . Si NBDCND toma cualquier otro valor, BDC es un parámetro mudo.

$\mathrm{BDD}=$ arreglo unidimensional de longitud $\mathrm{M}+1$ que especifica los valores de $\frac{D U\left(X_{i} . D\right)}{D Y}$, considerando $i=1, \ldots, M+1$, cuando NBDCND $=2$ ó 3 . Si NBDCND toma cualquier otro valor, BDD es un parámetro mudo.

COFX = es un subprograma proporcionado por el usuario, con parámetros $\mathrm{X}, \mathrm{AFUN}, \mathrm{BFUN}, \mathrm{CFUN}$, el cual regresa los valores de los coeficientes que dependen de $\mathrm{X}$ : $\mathrm{AF}(\mathrm{X}), \mathrm{BF}(\mathrm{X}), \mathrm{CF}(\mathrm{X})$, en la ecuación elíptica. GRHS = arreglo bidimensional que especifica los valores del lado derecho de la ecuación elíptica. En la frontera, GRHS, está definido como:

\begin{tabular}{|c|c|c|c|}
\hline MBDCND & $\operatorname{GRHS}(1, j)$ & $\operatorname{GRHS}(\mathrm{M}+1, \mathrm{j})$ & \\
\hline 0 & $G(A, Y j)$ & $G(B, Y j)$ & \\
\hline 1 & * & $*$ & \\
\hline 2 & $*$ & $G(B, Y j)$ & $j=1, \ldots, N+1$. \\
\hline 3 & $G(A, Y j)$ & $G(B, Y j)$ & \\
\hline 4 & $G(A, Y j)$ & * & \\
\hline
\end{tabular}

\begin{tabular}{|c|c|c|c|}
\hline NBDCND & GRHS $(i, 1)$ & $\operatorname{GRHS}(j, N+1)$ & \\
\hline 0 & $G(X i, C)$ & $G(X i, D)$ & \\
\hline 1 & $*$ & * & \\
\hline 2 & * & $G(X i, D)$ & $i=1, \ldots, M+1$. \\
\hline 3 & $G(X i, C)$ & $G(X i, D)$ & \\
\hline 4 & $G(X i, C)$ & $*$ & \\
\hline
\end{tabular}


$y$ en donde ${ }^{*}$ significa que estar cantidades no son usadas.

USOL $=$ arreglo bidimensional que especifica los valores de la solución en el interior y a lo largo de la frontera; en la frontera está definido por:
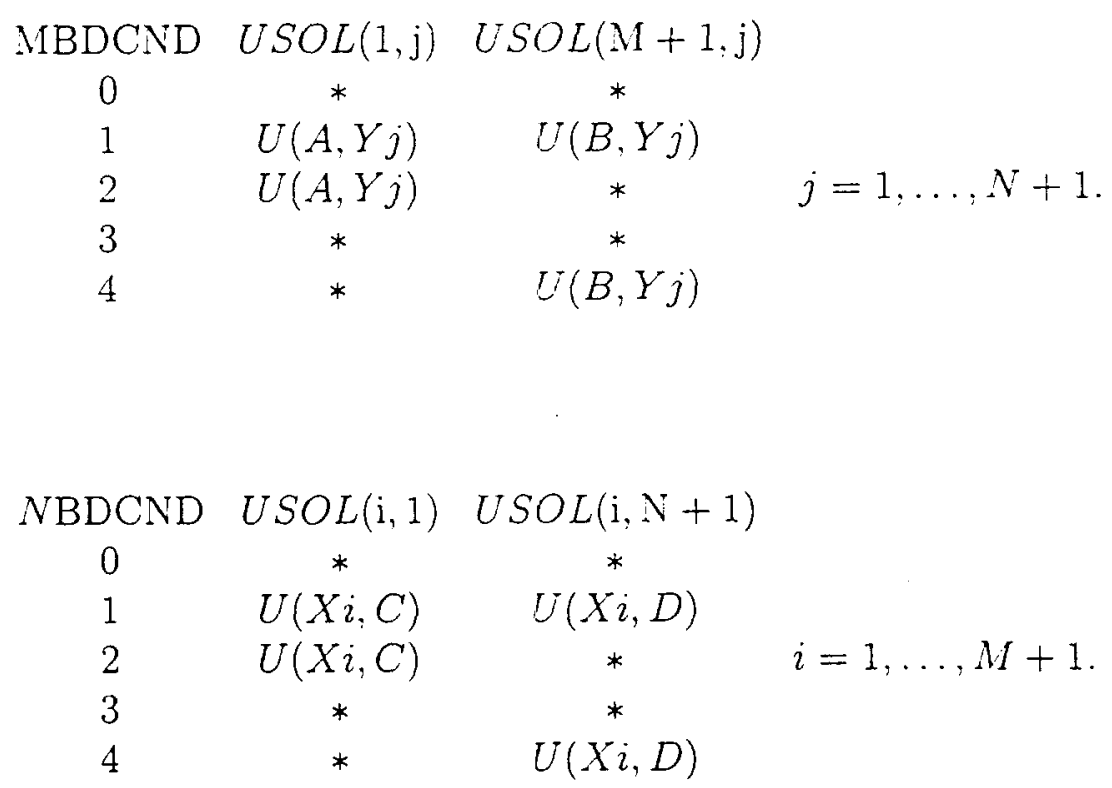

y en donde * significa que estas cantidades no son usadas en la solución. Si IORDER $\doteq 2$ USOL y GRHS pueden ser los mismos arreglos, pero si IORDER $=4$ los dos arreglos deben ser distintos. Además USOL debe estar dimensionada con al menos $\mathrm{N}+1$ en la rutina de llamado.

IDMN = Es usado para especificar el número de renglones de GRHS y de USOL. Debe ser al menos 7 y mayor o igual a $M+1$.

$W=$ Arreglo unidimensional que es usado como espacio de trabajo.

Como salida vamos a obtener: 
USOL $=$ Solución aproximada de la ecuación elíptica.

PERTRB $=$ Si se usa una combinación de condiciones de frontera en la derivada o periódicas y si $C F(X)=0$ para toda $X$, puede ser que no exista una solución. PERTRB es una constante que es calculada y sustraída del lado derecho, asegurando la existencia de una solución.DEPX4 calcula esta solución, la cual es una solución de mínimos cuadrados ponderados al problema original. Si no se detecta alguna singularidad, PERTRB = 0.0 .

IERROR = Bandera de error que indica la validez de los parámetros de entrada o falla para encontrar una solución:

$=0$ ningún error

$=1$ si $\mathrm{A}>\mathrm{B}$ o $\mathrm{C}>\mathrm{D}$

$=2$ si $\mathrm{MBDCND}<0$ ó $\mathrm{MBDCND}>4$

$=3$ si $\mathrm{NBDCND}<0$ ó NBDCND $>4$

$=4$ el intento por encontrar la solución falló

$=5 \mathrm{IDMN}$ es demasiado pequeño

$=6 \mathrm{M}$ es muy pequeño o muy grande

$=7 \mathrm{~N}$ es muy pequeño

$=8$ IORDER no es igual a 2 ó a 4

$=9$ INTL no es igual a 0 ó a 1

$=10$ AFUN $<=0$ para algún punto interior de la malla

$=11$ si la longitud del espacio de trabajo $\mathrm{W}$ no es suficiente. Esta longitud debe estar en $\mathrm{W}(1)$ antes del llamado

$=12$ si $\mathrm{MBDCND}=0$ y $\mathrm{AF}(\mathrm{X})=\mathrm{CF}(\mathrm{X})=$ constante o si $\mathrm{BF}(\mathrm{X})=0$ no se satisface para todo $\mathrm{X}$

Es importante hacer notar que el poder de este paquete radica por una parte, en el método de Reducción Cíclica [19] que usa para resolver eficientemente el sistema de ecuaciones resultante de la discretización, el cual es una variante del método de Buneman. Y por otro lado en que se puede hacer una aproximación de CUARTO ORDEN (IORDER $=4)$ a la solución de la ecuación a resolver. 


\section{Bibliografía}

[1] Batchelor, G. K. Heat transfer by free convection across a closed cavity between vertical boundaries at different temperatures, Quart. J. Appl, Math., 12, 209 (1954).

[2] Gill, A. E. The boundary layer regime for convection in a rectangular cavity, J. Fluid Mech., 26, 515 (1966).

[3] J. W. Elder. Transient convection in a poros medium ,J. Fluid Mech., 27, 609-623 (1967).

[4] Cormack, D. E., Leal, L. G. \& Imberger, J. Natural convection in a shallow cavity with differentially heated end walls , J. Fluid Mech., 65, 209 (1974).

[5] R. N. Horne and M. J. O'Sullivan. Oscillatory convection in a porous medium heated from below, J. Fluid Mech., 66, 339-352 (1974).

[6] Schubert, G. \& Straus, J. M. Three- dimensional and multicellular steady and unsteady convection in fluid-saturated porous media at high Rayleigh numbers , J. Fluid Mech., 94, 25 (1979).

[7] D. M. Christopher. Transient natural convection in a cavity filled with a porous medium, Heat Transfer in Porous Media and Particulate Flows, ASME Publication HTD 46, 191-198 (1985).

[8] D. M. Christopher. Transient natural convection heat transfer in a cavity filled with a fluid-saturated, porous medium, Preprint (1994).

[9] G. D. Mallinson and G. De Vahl Davis. Three-dimensional natural convection in a box: a numerical study, J. Fluid Mech., 83, 1-31 (1977). 
[10] B. Bermúdez and A. Nicolás. An Operator splitting numerical scheme for thermal/isothermal incompressible viscous flows, Int. J. Numer. Methods Fluids, 29, 397-410 (1999).

[11] H. Jiménez Islas. Modelamiento matemático de los procesos de transferencia de momentum, calor y masa en medios porosos, Tesis Doctoral, UAM-I, CBI, (1999).

[12] Batchelor, G.K. An introduction to fluid dynamics, Cambridge University.

[13] Bear, Jacob. Dynamics of fluids in porous media, American Elsevier Publication.

[14] Collins, R. E. Flow of fuids through porous materials, Pennwell publishing company.

[15] Dagan, Gedeon. Flow and transport in porous formations, SpringerVerlag pub.

[16] Landau, L. D. and Lifshitz, E. M. Fluid Mechanics. 2o. ed. Course of theoretical physics. 2o. ed. Vol. 6, Oxford, Pergamon Press.

[17] Turner, John S. Buoyancy effects in fluid, Cambridge University Press.

[18] Adams, John, Swarztrauber, Paul and Sweet, Roland FishPak. A package of Fortran subprograms for the solution of separable elliptic partial differential equations, The Nattional Center for Atmospheric Research Boulder, Colorado, U. S. A.

[19] Sweet, R., A cyclic reduction algorithm for solving block tridiagonal systems of arbitrary dimensions, SIAM J. on Numer. Anal., 14, 706-720, (1977). 